\title{
Porphyromonas gingivalis induces penetration of lipopolysaccharide and peptidoglycan through the gingival epithelium via degradation of coxsackievirus and adenovirus receptor
}

Hiroki Takeuchi ( $\nabla$ takeuchih@dent.osaka-u.ac.jp )

Osaka University

Shunsuke Yamaga

Osaka University

Naoko Sasaki

Osaka University

Masae Kuboniwa

Osaka University

Michiya Matsusaki

Osaka University

Atsuo Amano

Osaka University

Research Article

Keywords: Porphyromonas gingivalis, periodontal disease

Posted Date: February 23rd, 2021

DOl: https://doi.org/10.21203/rs.3.rs-181107/v1

License: (c) This work is licensed under a Creative Commons Attribution 4.0 International License.

Read Full License 
Porphyromonas gingivalis induces penetration of lipopolysaccharide and peptidoglycan through the gingival epithelium via degradation of coxsackievirus and adenovirus receptor

$$
\text { Atsuo Amano }{ }^{1 *}
$$

1 Department of Preventive Dentistry, Graduate School of Dentistry, Osaka University, Suita-Osaka 565-0871, Japan 2 Joint Research Laboratory (TOPPAN) for Advanced Cell Regulatory Chemistry, Graduate School of Engineering, Osaka University, Suita-Osaka 565-0871, Japan 3 Department of Applied Chemistry, Graduate School of Engineering, Osaka University, Suita-Osaka 565-0871, Japan

4 These authors have contributed equally to this work

\section{Short title: CXADR prevents LPS and PGN penetration through gingival epithelium}

*Address correspondence to:

Hiroki Takeuchi, DDS, PhD, Assistant Professor: e-mail: takeuchih@dent.osaka-u.ac.jp Atsuo Amano, DDS, PhD, Professor: e-mail: amanoa@dent.osaka-u.ac.jp

Department of Preventive Dentistry, Osaka University Graduate School of Dentistry, 1-8 Yamadaoka Suita-Osaka 565-0871, JAPAN

Tel: +81-6-6879-2921, Fax: +81-6-6879-2925 


\section{Abstract}

Porphyromonas gingivalis is a major pathogen in severe and chronic manifestations of periodontal disease, which is one of the most common infections of humans. A central feature of $P$. gingivalis pathogenicity is dysregulation of innate immunity at the gingival epithelial interface. We previously showed that junctional adhesion molecule 1 (JAM1) was specifically degraded by $P$. gingivalis, leading to epithelial barrier breakdown in gingival tissues. Whereas, the involvement of the other JAM family protein(s) in the epithelial barrier dysregulation by $P$. gingivalis remains unknown. Here we show that Arg-specific or Lys-specific cysteine proteases named gingipains produced by $P$. gingivalis specifically degrade coxsackievirus and adenovirus receptor (CXADR), a tight junction associated protein, at R145 and K235 in gingival epithelial cells. A $P$. gingivalis strain lacking gingipains was impaired in degradation of CXADR. Knockdown of CXADR in a three-dimensional multilayered tissue model increased permeability to $40 \mathrm{kDa}$ dextran, lipopolysaccharide, and proteoglycan. Inversely, overexpression of CXADR in a gingival epithelial tissue model prevented penetration by these agents following $P$. gingivalis infection. Our findings strongly suggest that $P$. gingivalis gingipains disrupt barrier function of stratified squamous epithelium via degradation of CXADR as well as JAM1, efficiently allowing bacterial virulence factors to penetrate into subepithelial tissues. 
Main

45

46

\section{Introduction}

Periodontal diseases are chronic infectious diseases caused by complex actions of periodontal bacteria in oral biofilm, and one of the most common infectious diseases of humans [1]. In periodontal tissues, the subgingival epithelium responds to microbial infection and harmonizing innate immunity [2]. Lipopolysaccharide (LPS: endotoxins of gram-negative bacteria) and peptidoglycan (PGN: mesh-like patterns outside the plasma membrane of most bacteria) are prototypal classes of pathogen-associated molecular patterns (PAMPs) that are recognized by the innate immunity [3].

The epithelium lining gingival sulcus is stratified squamous. Gingival epithelial cells express molecular complexes regulating cell-to-cell adhesion [4]. Human gingival epithelial cells express junctional adhesion molecules (JAMs), members of an immunoglobulin superfamily, that seal the paracellular pathway and prevent solutes passing through the paracellular space [4,5]. Homodimerization of JAMs is important for regulating epithelial barrier function [6]. However, barrier function of the sole JAM family proteins in human epithelium remains largely unknown.

Porphyromonas gingivalis, a keystone periodontal pathogen, can impair host immune defense and promote inflammation [7]. P. gingivalis secrets Arg-specific and Lysspecific cysteine proteases, termed Arg-gingipains (RgpA and RgpB) and Lys-gingipain (Kgp), respectively, which possess abundant proteolytic activities [8,9]. Hence, host proteins targeted and degraded by gingipains could be considered crucial factors influencing pathogenesis of periodontitis. We previously identified JAM1 as a gingival epithelial barrier protein, which is specifically degraded by $P$. gingivalis gingipains, leading to epithelial barrier breakdown by allowing increased epithelial permeability to 
PAMPs [10]. Whereas, the possibility that other JAM family proteins are targeted by $P$. gingivalis has not been verified. In this study, we screened the JAM family proteins involved in gingival epithelial barrier dysfunction by $P$. gingivalis using a threedimensional multilayered tissue model (3D-tissue model). We consequently identified CXADR as an additional JAM family protein targeted specifically by the pathogen. Gingipains degrades CXADR at R145 and K235, thereby inducing gingival epithelial permeability and allowing subsequent transmission of LPS and PGN. This work provides insights into process by which gingival epithelial barrier is subverted during periodontal pathogenesis.

\section{Results}

\section{P. gingivalis gingipains degrade CXADR in gingival epithelial cells}

To elucidate the JAM1-independent mechanism of gingival epithelial barrier function, we screened eight JAM family proteins [6] for their expression in immortalized human gingival epithelial (IHGE) cells. For this purpose, we used reverse transcriptional polymerase chain reaction (RT-PCR), and found that JAM2, CLMP, and CXADR were expressed in IHGE cells in addition to JAM1 (Fig. 1a). While, the negligible expression of JAM3, JAM4, CD2, and ESAM was observed in IHGE cells. Next, we investigated whether gingipains degrade these JAM family proteins cell at the endogenous protein level. We infected IHGE cells with P. gingivalis ATCC33277 wild type (WT) or its isogenic mutant KDP136 ( $\Delta \operatorname{kgp} \Delta \operatorname{rgp} A \Delta r g p B)$ for $1 \mathrm{~h}$ at a multiplicity of infection (MOI) of 100. We detected the decreased levels of JAM1, CXADR, and CLMP in IHGE cells were decreased by $P$. gingivalis WT, while the $\Delta \operatorname{kgp} \Delta \operatorname{rgp} A \Delta \operatorname{rgp} B$ mutant had a negligible effect (Fig. 1b). By contrast, P. gingivalis did not degrade JAM2 at $1 \mathrm{~h}$ after infection. 
These results suggest that gingipains specifically degrade JAM1, CXADR, and CLMP.

To assess the contribution of CXADR or CLMP expression to the permeability of gingival epithelial cells, we generated IHGE cell lines stably expressing small hairpin RNA (shRNA) against JAM1 (shJAM1 \#110 and shJAM1 \#508), CXADR (shCXADR \#38 and shCXADR \#317), and CLMP (shCLMP \#661 and shCLMP \#771), respectively. We then performed permeability assays using a small-molecule fluorescent probe (Fig. 2a). Knockdown of JAM1, CXADR, and CLMP in each cell line was confirmed by immunoblot (Fig. 2b). As shown in Figure 2c, IHGE monolayers expressing shRNA against CXADR as well as JAM1 were significantly more permeable to $40 \mathrm{kDa}$ FITCdextran, FITC-labeled $P$. gingivalis LPS, and FITC-labelled $P$. gingivalis PGN than control cells expressing shRNA against firefly luciferase (shLuc). By contrast, knockdown of CLMP had a negligible effect on the permeability to these tracers.

We examined the effects of secreted gingipains on CXADR in the same way that we previously studied the impact of gingipains on JAM1 [10]. The bacterial culture supernatant was collected and administered to IHGE cells for $1 \mathrm{~h}$. We detected the decreased levels of CXADR in IHGE cells by the culture supernatant of $P$. gingivalis WT. By contrast, the $\Delta \operatorname{kgp} \Delta \operatorname{rgp} A \Delta \operatorname{rgp} B$ mutant showed a negligible effect (Fig. S1). These results indicate that CXADR are degraded by secreted gingipains.

We next examined the association of $P$. gingivalis with CXADR in IHGE cells using a laser confocal microscope. At $1 \mathrm{~h}$ after infection of $P$. gingivalis WT the signal intensity of CXADR was reduced in the surface area of IHGE cells (Fig. 3a). By contrast, the $\Delta \operatorname{kgp} \Delta \operatorname{rgp} A \Delta \operatorname{rgpB}$ mutant failed to reduce the CXADR signal, consistent with the results in Figure $1 \mathrm{~b}$ and $1 \mathrm{c}$. In order to examine the effects of gingipains on CXADR degradation in deeper epithelium, we created a 3D-tissue model of IHGE cells 
116 by the cell-accumulation technique (Fig. 3b) [11]. We infected the 3D-tissue models

117 with $P$. gingivalis WT or $\Delta \operatorname{kgp} \Delta \operatorname{rgp} A \Delta \operatorname{rgp} B$, and analyzed the CXADR degradation in 118 deeper epithelium using confocal microscopy. At $2 \mathrm{~h}$ after infection, $P$. gingivalis WT 119 is capable of reducing the CXADR signal even in the tissues 3-4 layers below the surface 120 (Fig 3b). By contrast, the $\Delta \operatorname{kgp} \Delta \operatorname{rgp} A \Delta \operatorname{rgpB}$ mutant failed to reduce the CXADR signal. 121 These results suggest that gingipains deeply invade human gingival epithelial tissues and degrade CXADR.

CXADR is localized in the plasma membrane following cleavage of $\mathrm{N}$-terminal signal peptide

We hypothesized that $P$. gingivalis gingipains degrades CXADR localizes on the plasma membrane, but not efficiently in intracellular space. CXADR has a predicted signal peptide followed by immunoglobulin (IG) domains and a transmembrane domain $[12,13]$. In order to test this hypothesis, we visualized the intracellular localization of CXADR before and after cleavage of its signal peptide. We created a plasmid encoding Myctagged human influenza hemagglutinin (HA)-inserted CXADR (Fig. 4a) and examined

132 the localization of the chimeric protein in IHGE cells to determine by confocal microscopy. To monitor the CXADR localization, we stained IHGE cells expressing HA-tagged enhanced green fluorescent protein (EGFP) (Fig. S2a) or Myc-tagged HAinserted CXADR (Fig. S2b) using anti-HA antibody, with or without permeabilization. We were able to label HA-EGFP protein with anti-HA antibody in the permeabilized IHGE cells, but not in the non-permeabilized cells, suggesting that the cytosolic proteins

138 are not stained without permeabilization. By contrast, we were able to label HA139 inserted CXADR protein with anti-HA antibody even without permeabilization, 
suggesting that the ectopic protein of HA-inserted CXADR is properly transported to

141 plasma membrane.

142 In order to trace the pathway(s) of CXADR, we next analyzed IHGE cells expressing 143 Myc-tagged HA-inserted CXADR protein and each organelle marker: EGFP-SEC61 $\beta$ 144 (marker for endoplasmic reticulum membrane protein); TOMM20 (marker for outer 145 mitochondrial membrane protein; not included in endomembrane system) by confocal 146 microscopy. We detected co-localization of the anti-Myc and anti-HA signals with 147 SEC61 $\beta$ (Fig. 4b), but not TOMM20 (Fig. S3), suggesting that CXADR is transported 148 via an endomembrane pathway. Additionally, phalloidin-stained actin in the peripheral 149 area of IHGE cells co-localized with anti-HA-labeled CXADR, but not the Myc signal 150 (Fig. 4c). These results suggest that the signal peptide of CXADR is cleaved at the endoplasmic reticulum and transported to the plasma membrane, and that gingipains are capable of targeting mature form of CXADR at cell surface.

\section{P. gingivalis, but not $S$. gordonii or $F$. nucleatum, degrades CXADR}

155 In order to examine whether $P$. gingivalis degrades the immature and mature CXADR, 156 we infected IHGE cells expressing Myc-tagged HA-inserted CXADR, and the kinetics of Myc- and HA-tagged CXADR were analyzed. Considering the substrate specifically of a gingipains property, we utilized the HA tag as a marker of mature CXADR because $\mathrm{K}$ or $\mathrm{R}$ residues are not included in HA amino acid sequence (YPYDVPDYA). In addition, we distinguished immature and mature CXADR in immunoblots by the N-

161 terminal Myc tag. As shown in Figure 5a, P. gingivalis infection decreased the amount 162 of mature CXADR labeled with anti-HA, but failed to decrease on the level of immature 163 CXADR labeled with anti-Myc. These results suggest that mature form of CXADR are 
targeted by $P$. gingivalis. In order to examine the effects of another $P$. gingivalis strain, we infected IHGE cells with $P$. gingivalis TDC60 isolated from a severe periodontal lesion [14]. At 1 and $2 \mathrm{~h}$ after infection, P. gingivalis TDC60 markedly reduced mature from of CXADR (Fig 5b), indicating that other P. gingivalis strain is capable of degrading CXADR.

Human oral bacteria including Fusobacterium nucleatum and Streptococcus gordonii can establish mixed-species communities leading to periodontopathic biofilm formation. [15]. In order to examine the effects of $S$. gordonii or F. nucleatum on CXADR, we infected IHGE cells expressing Myc-tagged HA-inserted CXADR with these species. As shown in Figure 5c and 5d, S. gordonii and F. nucleatum fail to reduce the level of HA-inserted CXADR at $2 \mathrm{~h}$ after infection, suggesting that CXADR cannot be degraded by $S$. gordonii and F. nucleatum.

\section{CXADR R145 and K235 are responsible for degradation by $P$. gingivalis}

In order to examine which residue(s) are responsible for degradation by $P$. gingivalis gingipains, we constructed plasmids encoding deleted and mutated CXADR and performed a structural analysis (Fig. 6a). We transfected these plasmids into IHGE cells and then infected cells with $P$. gingivalis. To detect the residue(s) targeted by gingipains, we replaced the $\mathrm{R}$ and $\mathrm{K}$ residues with $\mathrm{H}$, a basic amino acid, in point mutation constructs. As shown in Figure 6b, degradation was observed in CXADR full length, CXADR $\triangle(1-$ 234), but not JAM1 $\Delta(1-234) \mathrm{K} 235 \mathrm{H}$, indicating that $\mathrm{K} 235$ is responsible for bacterial degradation. Additionally, the level of HA-tagged CXADR $\Delta(1-225) \mathrm{K} 235 \mathrm{H}$ and CXADR $\Delta(1-225) \mathrm{R} 226 \mathrm{H} \mathrm{K} 235 \mathrm{H}$ were not decreased by the infection, indicating that R226 is not responsible for bacterial degradation. Furthermore, the level of HA-tagged 
CXADR $\Delta(1-144) \mathrm{K} 235 \mathrm{H}$ was reduced following infection with $P$. gingivalis, but CXADR $\Delta(1-144)$ R145H K235H was not. These results illustrate that R145 and K235 are the residues targeted for degradation by gingipains.

CXADR prevents penetration of LPS and PGN through gingival epithelium

It is previously reported that CXADR mediates homotypic cell adhesion in Chinese hamster ovary $(\mathrm{CHO})$ cells [16]. However, the barrier function of CXADR in human squamous epithelium remains unknown. To assess the contribution of CXADR expression to the permeability of gingival deeper epithelium, we generated a 3D-tissue model stably expressing shLuc or shCXADR using the cell accumulation technique, and then performed permeability assays using a small-molecule fluorescent probe (Fig. 7a). Effective suppression of CXADR expression was confirmed by confocal microscopy (Fig. 7b). As shown in Figure 7c, IHGE monolayer expressing shRNA against CXADR was significantly more permeable to $40 \mathrm{kDa}$ FITC-dextran than control cells expressing shLuc. This result suggests that CXADR is involved in the kinetics of flux of molecules with 40 $\mathrm{kDa}$ between gingival epithelial cell layers.

We previously showed that JAM1 prevented permeation to dissociated LPS and PGN monomer ( $\mathrm{N}$-acetylglucosamine- $\mathrm{N}$-acetylmuramic acid) in the 3D-tissues of gingival epithelium [10]. This result prompted us to examine whether knockdown of CXADR allows permeation of LPS and PGN. Thirty minutes after administration, we detected the increased permeability to FITC-P. gingivalis LPS (Fig. 7d) and FITC-P. gingivalis PGN (Fig. 7e) by knockdown of CXADR. In order to confirm the generality of permeation of LPS and PGN following knockdown of CXADR, we also performed the permeability assay of Escherichia coli LPS and Staphylococcus aureus PGN through 
212 gingival epithelial tissues. In tissues expressing shCXADR, we detected the increased

213 permeability to FITC-E. coli LPS (Fig. 7d) and FITC-S. aureus PGN (Fig. 7e) at 30 min

214 after administration, suggesting that CXADR affects the permeability of gingival 215 epithelium to LPS and PGN.

216

\section{Epithelial barrier function is regulated by CXADR independently of JAM1}

As CXADR and JAM1 are of JAM family protein [6], these two proteins may interact

with each other to mediate its localization in surface of gingival epithelial cells. To assess the interdependence of CXADR and JAM1, we determined the localization of CXADR and JAM1 in IHGE cells expressing shCXADR \#317 or shJAM1 \#508 by confocal microscopy. As shown in Figure S4a, the localization of JAM1 in IHGE monolayer expressing shJAM1 was negligible, but was not disturbed in IHGE cells expressing shCXADR. In the same trend, the expression level of CXADR in IHGE monolayer expressing shCXADR was negligible, but was not disturbed in IHGE cells expressing shJAM1 (Fig. S4b). These results suggest that CXADR and JAM1 are not interdependent on localization in gingival epithelial cells. shJAM1 and the localiation of deeply seated CXADR and JAM1 was analyzed using confocal microscopy. The expression levels of IHGE cells expressing shCXADR and shJAM1 were confirmed by immunoblots (Fig. 8a). As shown in Figure 8b, shJAM1 caused JAM1, but not CXADR, to disappear even in the tissues 3-4 layers below the surface. In the same trend, shCXADR caused CXADR, but not JAM1, to disappear in the tissues below the surface. Finally, double knockdown of shCXADR and shJAM1 
caused these two proteins to disappear in the tissues. These results suggest that the localization of CXADR and JAM1 are not interdependent even in gingival epithelium model. As shown in Figure 8c, depletion of either CXADR or JAM1 enhances 239 permeability of gingival epithelium to $40 \mathrm{kDa}$ dextran, and depletion of both CXADR 240 and JAM1 showed an additional effect. These results show that CXADR mediates barrier function of gingival epithelium independently of JAM1.

CXADR degradation is involved in penetration of gingipains through gingival epithelial barrier

We previously showed that JAM1 degradation by gingipains is required for gingipains penetration in gingival epithelial cells [10]. In order to examine the CXADR-dependent mechanism of gingipains penetration, we employed a two-layered cell culture model, by which we can detect penetration of gingipains from the upper to the lower space (Fig. 9a). Six hours after administration of bacterial culture media, we confirmed the decreased level of HA-inserted CXADR, but not Myc-tagged CXADR, in the cells of the lower layer by $P$. gingivalis WT to a greater extent than by the $\Delta \operatorname{kgp} \Delta \operatorname{rgp} A \Delta \operatorname{rgp} B$ mutant (Fig. 9b). Next, we examined whether overexpression of CXADR, the expression level of which compensates CXADR degradation by $P$. gingivalis, blocks the loss of CXADR in gingival epithelial cells. To test this idea, we employed two-layered culture of IHGE cells overexpressing CXADR, and the localization of CXADR in cells of the lower layer were monitored following $P$. gingivalis infection. When IHGE cells overexpressing CXADR were treated with bacterial culture media of $P$. gingivalis for $30 \mathrm{~min}$, we detected the remaining CXADR proteins at almost the same level as in non-treated IHGE cells (Fig. S5), suggesting that degradation can be compensated by overexpression of CXADR 
in this system. In line with this result, $6 \mathrm{~h}$ after administration, we detected the increased

261

262

263

264

265

266

267

268

269

270

271

272

273

274

275

276

277

278

279

280

281

282

levels of HA-inserted CXADR in the lower layer when the cells in the upper layer overexpressed CXADR (Fig. 9b). These results indicate that degradation of CXADR by gingipains is important for penetration of the proteases through gingival epithelial cells.

\section{Degradation of CXADR by $P$. gingivalis causes penetration of LPS and PGN}

Next, we generated 3D-tissue model using IHGE cells overexpressing CXADR (Fig. 10a).

We confirmed that marked amounts of CXADR were remained even in tissues infected with P. gingivalis (Fig. 10b). In order to examine the permeability, the tissues were then treated with 40 kDa FITC-dextran, FITC-P. gingivalis LPS, or FITC-P. gingivalis-PGN. Thirty minutes after administration, we detected the decreased permeability to $40 \mathrm{kDa}$ FITC-dextran (Fig. 10c), FITC-P. gingivalis LPS (Fig. 10d), FITC-P. gingivalis PGN (Fig. 10e), FITC-E. coli LPS (Fig. 10f), and FITC-S. aureus PGN (Fig. 10g) in gingival epithelial tissues overexpressing CXADR. These results indicate that CXADR degradation by $P$. gingivalis is key for allowing the penetration of LPS and PGN in gingival epithelium.

\section{Discussion}

Our findings clearly suggest a molecular basis for the dysfunction of gingival epithelial barrier by $P$. gingivalis. Based on our results, we postulate the following model (Fig. 11). As CXADR as well as JAM1 localizes in a phalloidin-stained plasma membrane region in the 3D-tissue (Fig. 8b), CXADR and JAM1 function together as gingival epithelial barrier. By degrading not only JAM1 but also CXADR, P. gingivalis efficiently allows penetration of LPS, PGN, and gingipains derived from itself and other 
bacteria through the gingival epithelium and into deeper periodontal tissues. In this manner, $P$. gingivalis is probably able to destroy the epithelial barrier, which remotely causes alveolar bone loss.

In this study, we employed a 3D-tissue model using the cell-accumulation technique by a single cell coating with fibronectin/gelatin nanofilms [11]. We were able to generate gingival epithelium even with knockdown of CXADR and JAM1 (Fig. 8b), indicating that CXADR and JAM1 are not necessary for reconstruction of gingival epithelium. Hence, the cell-accumulation technique is likely useful for defining function of JAMs in the other types of epithelium.

We also generated HA-inserted CXADR for use as a probe in gingival epithelial cells. Using an HA-tagged protein, we were able to detect the responsible amino acid residues to bacterial degradation. P. gingivalis gingipains degrade CXADR at K235 (Fig. 6), and JAM1 at R234 [10]. The hinge region between the C-terminal IG-like domain and the transmembrane domain, possessing CXADR K235 and JAM1 R234, does not contain a consensus sequence for $\mathrm{N}$-glycosylation or secondary structure including helix, $\beta$-sheet, and turn (Uniplot, JAM1: https://www.uniprot.org/uniprot/Q9Y624; CXADR: https://www.uniprot.org/uniprot/P78310). Considering that the N-terminal IG-like domain of CXADR and JAM1 is responsible for homodimerization [21,22], gingipains effectively separate the $\mathrm{N}$-terminal region away from the transmembrane domain. gingival epithelial tissues to $40 \mathrm{kDa}$ dextran almost at the same level (Fig. 8c). Additionally, double knockdown of CXADR and JAM1 in gingival epithelial tissues 306 showed further increase of permeability compared to single knockdown (Fig. 8c). Upregulated CXADR or JAM1 messenger RNA expression is inhibited by the AP-1 or 
the NF- $\kappa \mathrm{B}$ inhibitor, respectively $[23,24]$. These multiple transcription pathways of CXADR and JAM1 may provide gingival epithelium a robust defense against external bacterial stress. Gingival epithelial function to control the barrier permeability is a

311 perspective that should be further investigated in the etiology of periodontal disease.

312 Based on the results of this study, there remain at least two questions that we should consider in the future. One is whether other major pathogens could specifically degrade

314 CXADR and JAM1. The increase in the amount of the "red complex" species $(P$. gingivalis, Treponema denticola, and Tannerella forsythia) in subgingival biofilms has been shown to be highly associated with the initiation and progression of periodontitis [17]. T. denticola expresses the serine protease, dentilisin and oligopeptidase B $[18,19]$. T. forsythia expresses the cysteine protease, forsythia detachment factor [20]. The effects of these proteases on CXADR and JAM1 will be further examined.

The other one is the relationship between protein structure and the degradation. We showed that CXADR and JAM1, but not Claudin (CLDN) 1 and CLDN 4, were degraded by $P$. gingivalis in gingival epithelial cells [10]. JAM family proteins possess a single transmembrane domain, while CLDN family proteins are of a tetra spans transmembrane protein and possess two extracellular loops [5]. Accordingly, it is necessary to study other gingipains-degradative proteins which potentially regulates gingival epithelial barrier without an extracellular loop. 


\section{Bacteria and cell culture}

P. gingivalis ATCC 33277 (purchased from the American Type Culture Collection), TDC60 (kindly provided by Kazuyuki Ishihara, Tokyo Dental College), and KDP136 ( $\Delta \operatorname{kgp} \Delta \operatorname{rgp} A \Delta \operatorname{rgp} B$, kindly provided by Koji Nakayama, Nagasaki University) [25] were maintained anaerobically on blood agar plates (BD) and grown in trypticase soy broth (BD), supplemented with hemin $\left(5 \mu \mathrm{g} \mathrm{mL} L^{-1}\right.$; WAKO $)$, and menadione $\left(1 \mu \mathrm{g} \mathrm{mL}^{-1}\right.$; Sigma-

Aldrich). S. gordonii ATCC 35105 were grown aerobically at $37{ }^{\circ} \mathrm{C}$ in Todd-Hewitt broth. F. nucleatum subsp. nucleatum ATCC 25586 were grown anaerobically at $37^{\circ} \mathrm{C}$ on blood agar plates (BD). IHGE cells (kindly provided by Shinya Murakami, Osaka University) were maintained in Humedia KG-2 (Kurabo), as described previously [26]. For the preparation of the bacterial culture supernatant of $P$. gingivalis, the bacterial culture at a stationary growth phase was centrifuged at 3,300 $\mathrm{g}$ for $3 \mathrm{~min}$, and the supernatant was collected and administered into the culture media of IHGE cells at a ratio of 1:50.

\section{Reverse transcriptional PCR}

347 Total RNA was extracted from IHGE cells using TRIzol (Thermo Fisher Scientific). 348 Complementary DNA was synthesized using the iScript cDNA Synthesis Kit (Bio-Rad). 349 Reverse transcriptional PCR was performed with the primers as follows: JAM1 forward, 5'-GTGCCTTCAGCAACTCTTCC-3'; reverse, 5'-ACCAGATGCCAAAAACCAAG- 
354 JAM4 forward, 5'-TCAAAGTGCAGTGAGCATCC-3'; reverse, 5'355 CCCCAAGTAGGCAATGAAAA-3'. CD2 forward, 5'356 CAGCCTGAGTGCAAAATTC-3'; reverse, 5'-CTCTGTGGGCTCTTGTCTCC-3'. 357 CLMP forward 5'-CAGGAGCAGTGACAGGCATA-3'; $\quad$ reverse, $\quad$ 5'358 TATTTGCTGTGGAGCGAGTG-3'. 359 GCCCTTTGTGATGTGGTTCT-3'; reverse, 5'-TTGCCTTGTTTGTCTTGCAC-3'. 360 CXADR forward, 5'-ATGCCCACTTCATGGTTAGC-3'; $\quad$ reverse, 5'GCGCTAGAGCAAGCAAAGTT-3'.

\section{Cell accumulation technique}

Three-dimensional culture of IHGE cells was performed as described previously [10]. IHGE cells collected by centrifugation after trypsinization were alternatively incubated for $1 \mathrm{~min}$ with $0.2 \mathrm{mg} \mathrm{mL}^{-1}$ fibronectin (Sigma-Aldrich) and $0.1 \%(\mathrm{w} / \mathrm{v})$ gelatin (Nacalai Tesque) dissolved in phosphate-buffered saline (PBS). After each procedure, cells were washed with PBS $(\mathrm{pH}=7.4)$ by centrifugation at $180 \mathrm{~g}$ for $2 \mathrm{~min}$ to remove unabsorbed polymers. After nine steps of immersion, fibronectin/gelatin nano-films were coated onto single-cell surfaces. A total of $2 \times 10^{6}$ cells coated with fibronectin/gelatin nanofilms were seeded on coverslips coated with $0.2 \mathrm{mg} \mathrm{mL}^{-1}$ fibronectin dissolved in PBS in 24-well plates (Iwaki). After $36 \mathrm{~h}$ of incubation, tissues were subjected to experiments, fixed, and analyzed on a confocal microscope (TCS SP8; Leica Microsystems). The number of $P$. gingivalis cells in culture media were adjusted to an optical density at 600 $\mathrm{nm}$ of 0.12 to infect gingival epithelial tissues. 
Mouse monoclonal anti-c-Myc (M4439), mouse monoclonal anti-HA (H3663), mouse monoclonal anti-JAM1 (SAB4200468), and mouse monoclonal $\beta$-ACTIN (A2228) antibodies were from Sigma-Aldrich; rabbit monoclonal anti-CXADR (10799-R271) was from Sino Bilogical; rabbit polyclonal anti-JAM2 (12972-1-AP) and rabbit polylonal anti-CLMP (16127-1-AP) was from Proteintech; rabbit monoclonal anti-HA antibody (3724) was from Cell Signaling Technology; rabbit monoclonal anti-TOMM20 (ab78547) was from Abcam. FITC-conjugated secondary (goat anti-mouse IgG, A-11001) antibody (MBL), and Alexa Fluor 488-conjugated secondary (goat anti-rabbit IgG, A11008), Alexa Fluor 568-conjugated secondary (goat anti-mouse IgG, A-11004), and Alexa Fluor 635-conjugated secondary (goat anti-rabbit IgG, A-31576) antibodies (Invitrogen) were used for fluorescence microscopy. Goat anti-mouse (7076) and antirabbit (7074) antibodies conjugated to horseradish peroxidase (Cell Signaling Technology) were used for immunoblotting. 4',6-diamidino-2-phenylindole (DAPI) (D1306, Invitrogen), Alexa Fluor 568- and Alexa Fluor 633-conjugated phalloidin (A12380 and A22284, respectively, Invitrogen) were used to stain IHGE cells.

\section{Plasmids}

The plasmid encoding Myc-tagged HA-inserted CXADR was constructed by cloning PCR products amplified from IHGE cells into pCMV-Myc (Clontech), using exogenously added EcoRI and KpnI sites. To produce HA-inserted CXADR, DNA sequence of HAtag was inserted into $C X A D R$ with fusion PCR. Plasmids encoding HA-tagged CXADR deletion mutants and point mutations were constructed by PCR. The plasmid encoding EGFP-SEC61 $\beta$ was constructed by cloning PCR products amplified from IHGE cells into pEGFP-C1 (Clontech). PCR amplification was performed with KOD Plus Neo 
402 (Kurabo). PCR products were ligated into plasmids with T4 DNA ligase (New England

403 Biolabs). Transfection of IHGE cells was performed using FuGENE 6 Transfection

404 Reagent (Promega).

405

$406 \quad$ Immunoblotting

407 Immunoblotting was performed as described previously [10]. IHGE cells were lysed, 408 and the lysates were cleared by centrifugation. Proteins were separated by SDS-PAGE 409 and transferred to nitrocellulose membranes $(0.2 \mu \mathrm{m}$, Bio-Rad). Membranes were 410 blocked for $1 \mathrm{~h}$ at room temperature with PBST (PBS and $0.1 \%(\mathrm{v} / \mathrm{v})$ Tween 20) 411 containing 3\%(w/v) skim milk, and then incubated for $1 \mathrm{~h}$ at room temperature with 412 primary antibodies diluted in PBST. Next, the membranes were washed three times with 413 PBST and incubated for $1 \mathrm{~h}$ at room temperature with 1:5000 dilutions of HRP414 conjugated secondary antibodies in PBST. Immunoreactive bands were detected using the Pierce ECL Western Blotting Substrate (Thermo Scientific) and ChemiDoc XRS (BioRad). Images were acquired using the Quantity One software (Bio-Rad).

\section{Immunocytochemistry}

419 Immunocytochemistry was performed as described previously [10]. IHGE cells were 420 fixed with 4\% (v/v) paraformaldehyde in PBS (Wako) overnight at room temperature, permeabilized with $0.1 \%(\mathrm{v} / \mathrm{v})$ Triton X-100 (Wako) in PBS for $5 \mathrm{~min}$ at room temperature, and blocked with $0.1 \%(\mathrm{w} / \mathrm{v})$ gelatin (Wako) in PBS for $20 \mathrm{~min}$ at room temperature. Samples were incubated for $1 \mathrm{~h}$ at room temperature with the indicated 424 primary antibodies, washed four times in PBS, incubated for $1 \mathrm{~h}$ at room temperature with the Alexa Fluor-conjugated secondary antibodies (Invitrogen), and again washed four 
times in PBS. All antibodies were diluted 1:400 in PBS. Cells were mounted onto glass slides with Vectashield Mounting medium (Vector Laboratories). Images were acquired with a confocal laser microscope (TCS SP8; Leica Microsystems) using a 64× oil-immersion object lens with a numerical aperture of 1.4. Acquired images were analyzed using the Application Suite X software (Leica Microsystems).

431

\section{Generation of cell line stably expressing CXADR}

Plasmid encoding CXADR was constructed by cloning PCR products amplified from the pCMV plasmid into pMRX-IRES-Puro (kindly provided by Nobumichi Furuta, Osaka University). Plasmids pMRX-IRES-Puro-CXADR was used for overexpression of the cDNA in IHGE cells. IHGE cells were transfected with the overexpression plasmid using FuGENE 6 (Promega). Seventy-two hours after transfection, cells stably overexpressing the cDNA were selected with puromycin $\left(2 \mu \mathrm{g} \mathrm{mL} \mathrm{mL}^{-1}\right.$; InvivoGen).

\section{RNA interference}

Plasmid encoding shRNA was constructed by ligation of linear DNA (Sigma-Aldrich) into pSIREN-RetroQ (631526, Clontech) or pSINsi-hU6 (3661, Takara). Plasmids pSIREN-RetroQ-shJAM1 $\# 110$ (target sequences: 5'AAGTCAGAATTCCTGAGAATAAT-3') and \#508 (target sequences: 5'GGGATAGTGATGCCTACGAATCC-3’), pSINsi-hU6-shJAM1 \#508 (Fig. 9), shCXADR \#38 (target sequences: 5'-TAGTGGATTTCGCCAGAAGTTTG-3') and \#317 447 (target sequences: 5'-ATGTAACGAATTTACAACTGTCA-3'), and shCLMP \#661 (target sequences: 5'-GTGCGAGTAACTGTACAGTATGT-3') and \#771 (target sequences: 5'-AAGGAAAGACAAAGAAAGATATG-3') were used for generation of 
the siRNA duplex in cells. Plasmid pSIREN-RetroQ-shLuc was produced as described previously [10]. IHGE cells were transfected with the shRNA-encoding plasmid using FuGENE 6 (Promega). Seventy-two hours after transfection, cells stably expressing the shRNA were selected with puromycin $\left(2 \mu \mathrm{g} \mathrm{mL} L^{-1}\right)$ and neomycin $\left(200 \mu \mathrm{g} \mathrm{mL}^{-1}\right.$; Invivogen)

\section{Preparation of FITC-labeled tracer}

Preparation of FITC-labeled tracer was performed as described previously [10]. $P$. gingivalis LPS (14F18-MM) was purchased from Invivogen. P. gingivalis PGN was prepared as described previously [27]. FITC-E. coli LPS (L7018) and S. aureus PGN (77140) were purchased from Sigma-Aldrich. Bacterial LPS and PGN were labeled with FITC using Fluorescein Labeling Kit-NH2 (LK-01, Dojindo). To generate dissociated LPS and PGN, FITC-labeled LPS was diluted by $10 \%$ with water and incubated with $10 \mathrm{mM}$ citrate (Wako) and 0.05\% (v/v) Tween-20 (Calbiochem) for 45 min at $37^{\circ} \mathrm{C}$ as described previously [28], and FITC-labeled PGN was diluted by $10 \%$ with water and incubated with $0.5 \mathrm{mg} \mathrm{mL}^{-1}$ lysozyme (Nacalai Tesque) for $45 \mathrm{~min}$ at $37^{\circ} \mathrm{C}$ to make a suspension. $40 \mathrm{kDa}$ FITC-dextran (FD40, Sigma-Aldrich) were diluted by $2 \%$ with water for the permeability assay.

\section{Epithelial barrier function assay}

Epithelial barrier function assay was performed as described previously [10]. The in vitro epithelial permeability assay to assess barrier function was performed with 12-well cell culture inserts (353180; Corning). When IHGE cells in the upper compartment reached $100 \%$ confluence, $20 \mu \mathrm{L}$ of FITC-dextran, FITC-LPS, or FITC-PGN was added 
to the upper compartment of the insert. After a 30-min incubation, the medium was

475

476

477

478

479

480

481

482

483

484

485

486

487

488

489

490

491

492

493

494

495

496

497

collected from the lower compartment, and the fluorescence intensity was measured using 1420 ARVO $X$ (PerkinElmer). Data were acquired using the WorkOut Plus software (PerkinElmer).

\section{Statistical analysis}

p-values were determined by $t$ test in Excel (Microsoft); $\mathrm{p}<0.05$ was considered significant.

\section{Acknowledgements}

We thank the Center for Oral Science, Graduate School of Dentistry, Osaka University for technical support with confocal laser microscopy. This research was supported by Grants-in-Aid for Young Scientists (B), Grant Numbers 17K17083 (to H.T.) and Scientific Research (C), Grant Numbers 19K10085 (to H.T.), and Scientific Research (A), Grant Numbers 18H04068 (to A.A.), from the Japan Society for the Promotion of Science. The funders had no role in study design, data collection, decision to publish, or preparation of the manuscript.

\section{Author Contributions}

H.T. and A.A. conceived and designed the experiments. H.T. and S.Y. performed the experiments. H.T. and A.A. analyzed the data. H.T., N.S., M.K., and M.M. contributed reagents, materials, and analytical tools. H.T. and A.A. wrote the paper.

\section{Competing Interests}


498 The authors have no competing financial interests to declare.

499 
501 1. Eke, P. I., Dye, B. A., Wei, I., Thornton-Evans, G. O. \& Genco, R. J. Prevalence of 502 periodontitis in adults in the United States: 2009 and 2010. J Dent Res. 91, 914-920 503 (2009).

2. Dixon, D. R., Bainbridge, B. W. \& Darveau, R. P. Modulation of the innate response within the periodontium. Periodontol 2000. 35, 53-74, (2004).

507

508

3. Kawai, T. \& Akira, S. Toll-like receptors and their crosstalk with other innate receptors in infection and immunity. Immunity. 34, 637-650 (2001).

510

4. Belibasakis, G. N., Kast, J. I., Thurnheer, T., Akdis, C. A. \& Bostanci, N. The expression of gingival epithelial junctions in response to subgingival biofilms. Virulence. 6, 704-709 (2005).

514

5. Tsukita, S., Furuse, M. \& Itoh, M. Multifunctional strands in tight junctions. Nat Rev Mol Cell Biol. 2, 285-293 (2001).

517

6. Ebnet, K., Suzuki, A., Ohno, S. \& Vestweber, D. Junctional adhesion molecules (JAMs): more molecules with dual functions? J Cell Sci. 117, 19-29 (2004).

7. Lamont, R. J., Koo, H. \& Hajishengallis, G. The oral microbiota: dynamic communities and host interactions. Nat Rev Microl. 16, 745-759 (2018).

8. Potempa, J., Pike, R. \& Travis, J. The multiple forms of trypsin-like activity present in various strains of Porphyromonas gingivalis are due to the presence of either Arggingipain or Lys-gingipain. Infect Immun. 63, 1176-1182 (1995).

9. Nakayama, K., Kadowaki, T., Okamoto, K. \& Yamamoto, K. Construction and characterization of arginine-specific cysteine proteinase (Arg-gingipain)-deficient mutants of Porphyromonas gingivalis. Evidence for significant contribution of Arg- 
531

532

533

534

535

536

537

538

539

540

541

542

543

544

545

546

547

548

549

550

551

552

553

554

555

556

557

558

559

560

561

562

gingipain to virulence. J Biol Chem. 270, 23619-23626 (1995).

10. Takeuchi, H., Sasaki, N., Yamaga, S., Kuboniwa, M. \& Amano, A. Porphyromonas gingivalis induces penetration of lipopolysaccharide and peptidoglycan through the gingival epithelium via degradation of junctional adhesion molecule 1. PLoS Pathog. 15, e1008124; 10.1371/journal.ppat.1008124 (2019).

11. Nishiguchi, A., Yoshida, H., Matsusaki, M. \& Akashi, M. Rapid construction of threedimensional multilayered tissues with endothelial tube network by the cell-accumulation technique. Adv Mater. 23, 3506-3510 (2011).

12. Bergelson, J. M. et al. Isolation of a common receptor for coxsackie B virus and adenoviruses 2 and 5. Science 275,1320-1323 (1997).

13. Tomko, R.P., Xu, R. \& Philipson, L. HCAR and MCAR: the human and mouse cellular receptors for subgroup C adenoviruses and group B coxsackieviruses. Proc. Natl. Acad. Sci USA. 94, 3352-3356 (1997).

14. Watanabe, T. et al. Complete genome sequence of the bacterium Porphyromonas gingivalis TDC60, which causes periodontal disease. J Bacteriol. 193, 4259-4260 (2011).

15. Kolenbrander, P. E. et al. Communication among oral bacteria. Microbiol Mol Biol Rev. 66, 486-505 (2002).

16. Cohen, C. J. et al. The coxsackievirus and adenovirus receptor is a transmembrane component of the tight junction. Proc Natl Acad Sci U S A. 98, 15191-15196 (2001).

17. Socransky, S. S., Haffajee, A. D., Cugini, M. A., Smith, C. \& Kent, R. L. Jr. Microbial complexes in subgingival plaque. J Clin Periodontol. 25, 134-144 (1998).

18. Ishihara, K., Miura, T., Kuramitsu, H. K. \& Okuda, K. Characterization of the Treponema denticola prtP gene encoding a prolyl-phenylalanine-specific protease 
19. Fenno, J. C., Lee, S. Y., Bayer, C. H. \& Ning Y. The opdB locus encodes the trypsin-

like peptidase activity of Treponemma denticola. Infect Immun. 69, 6193-6200 (2001).

567

20. Nakajima, T. et al. Isolation and identification of a cytopathic activity in Tannerella forsythia. Biochem Biophys Res Commun. 351, 133-139 (2006).

570

21. Patzke, C. et al. The coxsackievirus-adenovirus receptor reveals complex homophilic and heterophilic interactions on neural cells. J Neurosci. 30, 2897-2910 (2010).

22. Mandell, K. J., McCall, I. C. \& Parkos, C. A. Involvement of the junctional adhesion molecule-1 (JAM1) homodimer interface in regulation of epithelial barrier function. $J$ Biol Chem. 279, 16254-16262 (2004).

23. Azari, B. M. et al. Transcription and translation of human F11R gene are required for an initial step of atherogenesis induced by inflammatory cytokines. $J$ Transl Med. 9, 98; 10.1186/1479-5876-9-98 (2011).

581

24. Chung, J. et al. Coxsackievirus and adenovirus receptor mediates the responses of 583 endothelial cells to fluid shear stress. Exp Mol Med. 51, 1-15 (2019).

584

25. Shi, Y. et al. Genetic analysis of proteolysis, hemoglobin binding, and 586 hemagglutination of Porphyromonas gingivalis. Construction of mutants with a combination of rgpA, rgpB, kgp, and hagA. J Biol Chem. 274, 17955-17960 (1999).

26. Murakami, S. et al. Activation of adenosine-receptor-enhanced iNOS mRNA expression by gingival epithelial cells. J Dent Res. 81, 236-240 (2002). of the innate immune system in silkworm larvae. J Biol Chem. 285, 33338-33347 (2010). 
595 28. Reich, J., Lang, P., Grallert, H. \& Motschmann, H. Masking of endotoxin in surfactant 596 samples: Effects on Limulus-based detection systems. Biologicals. 44, 417-422 (2016). 597 598 
a
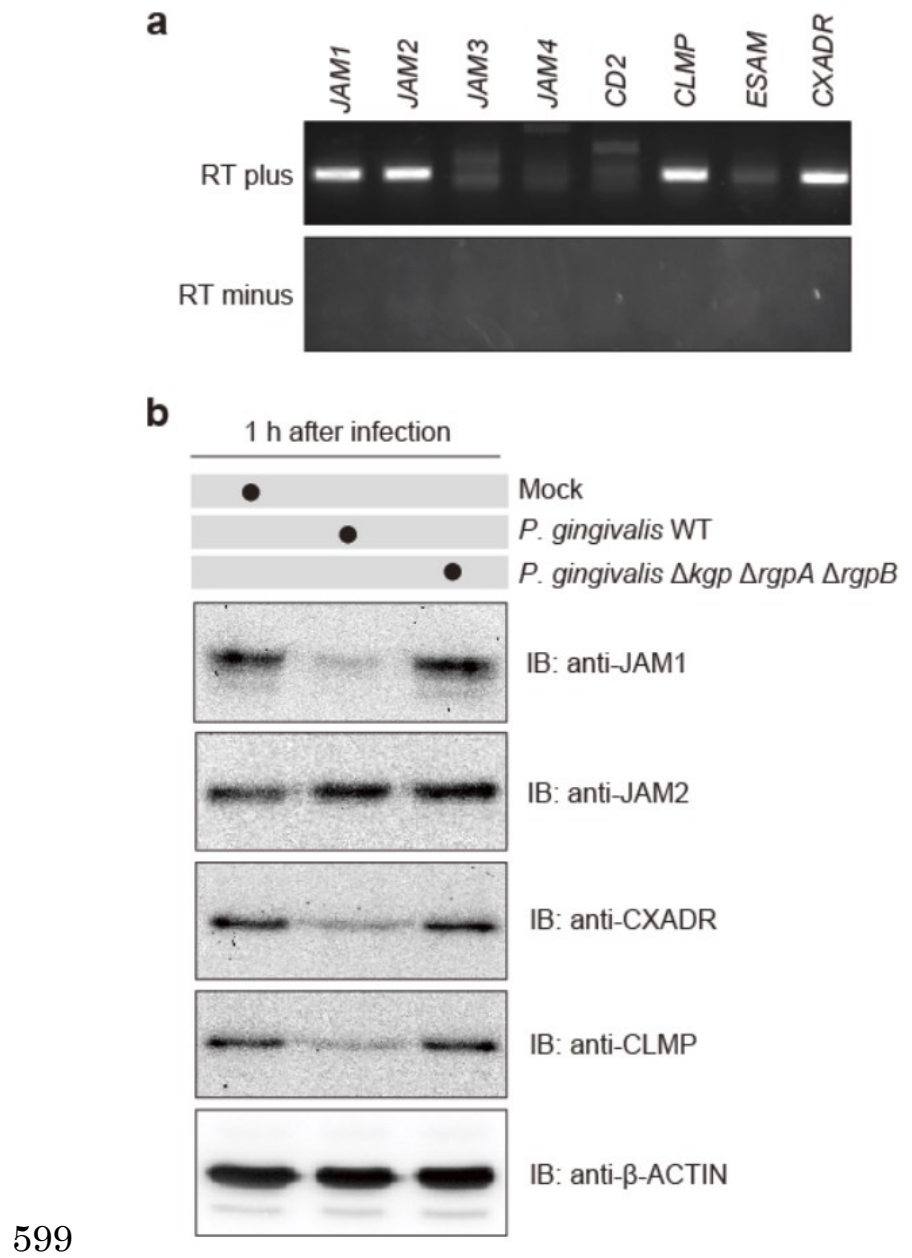

600

601 Figure 1. P. gingivalis gingipains degrade CXADR in IHGE cells.

602 (a) Expression of $J A M 1, J A M 2, J A M 3, J A M 4, C D 2, C L M P, E S A M$, and CXADR by IHGE 603 cells analyzed by RT-PCR. (b) IHGE cells were infected with $P$. gingivalis WT or $\Delta$ kgp $604 \Delta \operatorname{rgp} A \operatorname{sgp} B$ mutant at an MOI of 100 for $1 \mathrm{~h}$. The cells were then analyzed by 605 immunoblotting with the indicated antibodies. $\quad \beta$-ACTIN was used as a loading control. 606 RT, reverse transcription. IB, immunoblot. 
a

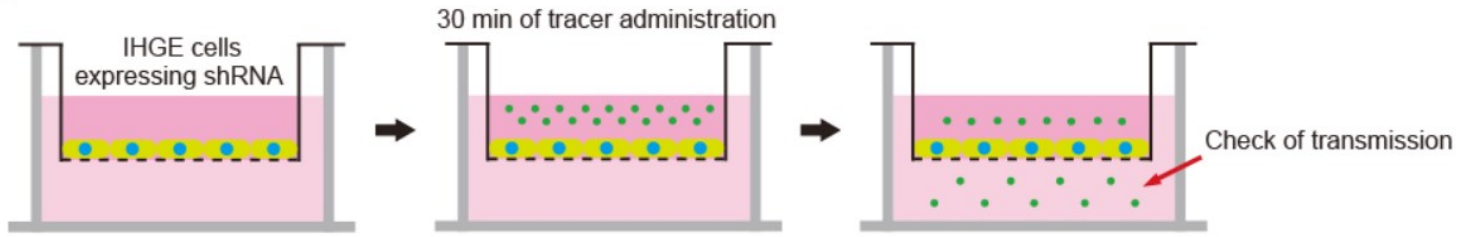

b

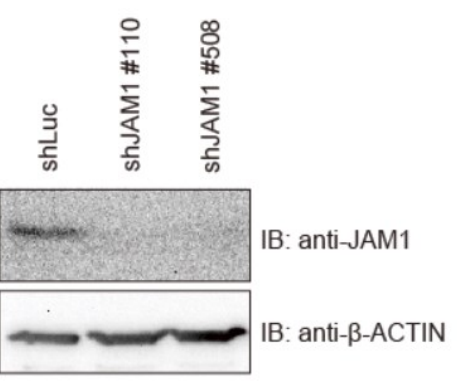

C

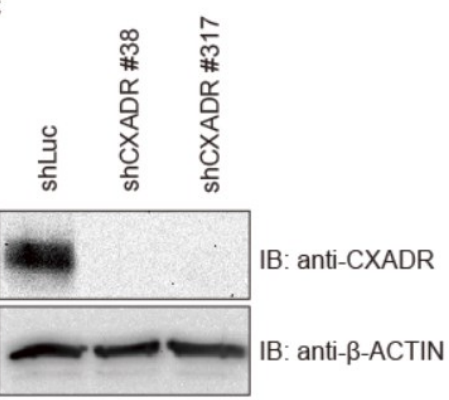

d

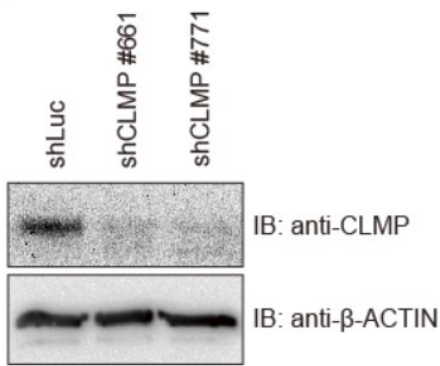

f

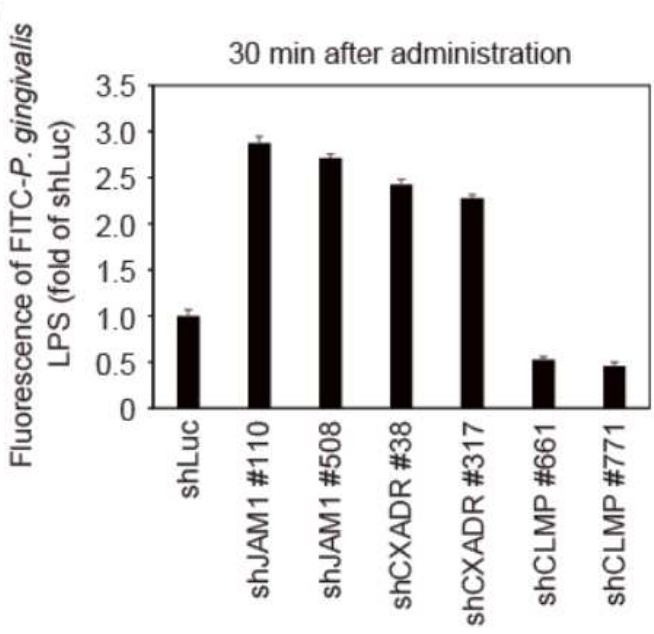

g
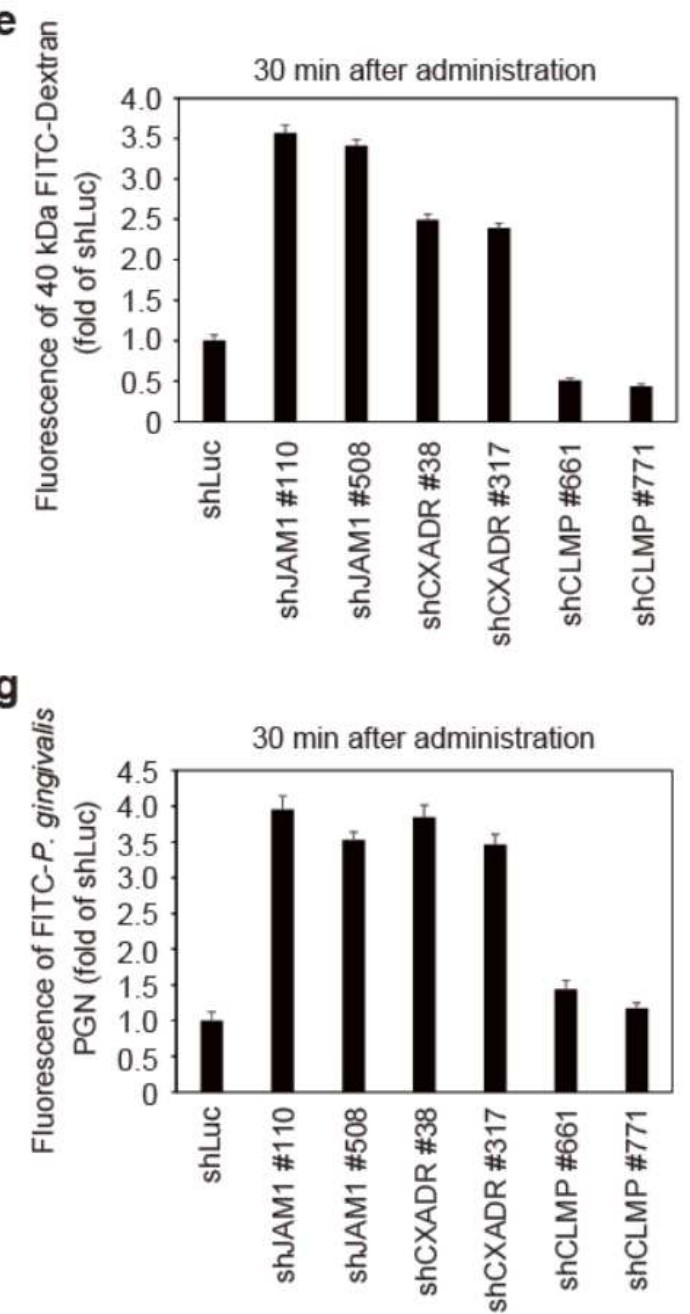

609

610

611 Figure 2. Loss of CXADR increased the epithelial barrier permeability. 
612 (a) Schematic image of the culture insert system. Monolayer of IHGE cells stably 613 expressing shLuc, shJAM1 \#110, shJAM1 \#508, shCXADR \#38, shCXADR \#317, 614 shCLMP \#661, and shCLMP \#771 were cultured in culture inserts. FITC-labeled tracer 615 was added to culture media in the upper compartment. Following 30 min of incubation, 616 the transmission of tracer from the upper compartment to the lower compartment was 617 analyzed by spectrometry. (b-d) IHGE cells stably expressing shLuc, shJAM1, 618 shCXADR, or shCLMP were analyzed by immunoblotting with the indicated antibodies. 619 (e-f) Permeability to $40 \mathrm{kDa}$ FITC-dextran (e), FITC- $P$. gingivalis LPS (f), FITC- $P$. 620 gingivalis PGN (g) in IHGE cells expressing indicated shRNA. Results are expressed 621 as fold change relative to cells expressing shLuc and are the means \pm SD of eight technical 622 replicates.

623

624

625 
a

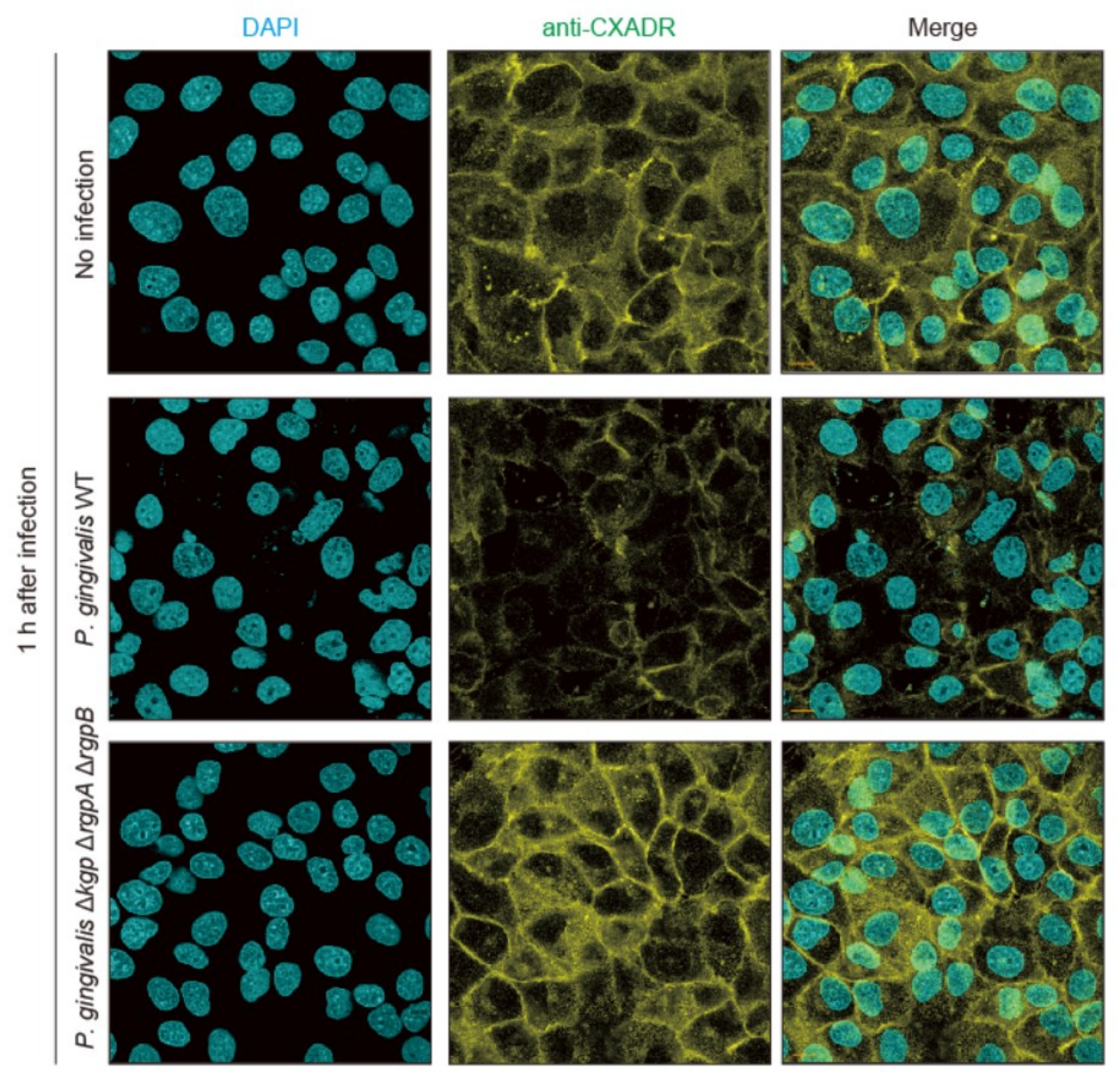

626

b
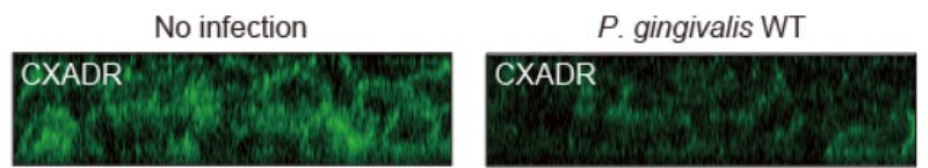

P. gingivalis $\triangle$ kgp $\triangle r g p A \triangle r g p B$
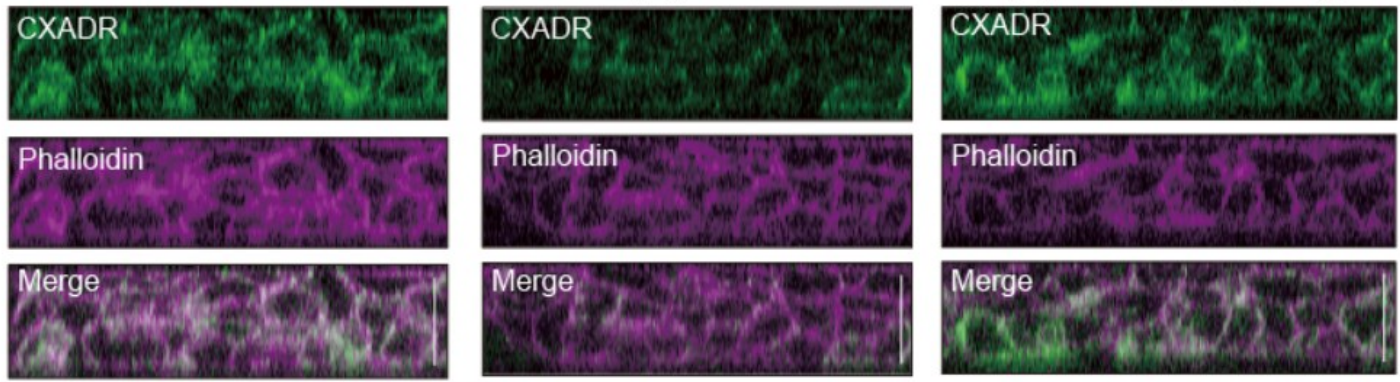

628

629 Figure 3. Degradation of CXADR in IHGE cells infected with $P$. gingivalis

630 gingipains

631 (a) IHGE cells were infected with $P$. gingivalis WT or $\Delta \operatorname{kg} p \Delta \operatorname{rgp} A \Delta \operatorname{rgp} B$ mutant at an

632 MOI of 100 for $1 \mathrm{~h}$. The cells were then fixed, stained with DAPI (cyan) and anti- 
633 CXADR (yellow), and analyzed by confocal microscopy. Scale bars, $10 \mu \mathrm{m}$.

634 Gingival epithelial tissues on coverslips were infected with $P$. gingivalis WT or $\Delta k g p$ $635 \Delta \operatorname{rgp} A \triangle \operatorname{rgp} B$ mutant for $2 \mathrm{~h}$. The tissues were then fixed, stained with anti-CXADR 636 (green) and Alexa Fluor 568-conjugated phalloidin (magenta), and analyzed by confocal 637 microscopy. Scale bars, $30 \mu \mathrm{m}$.

638

639

640 
a
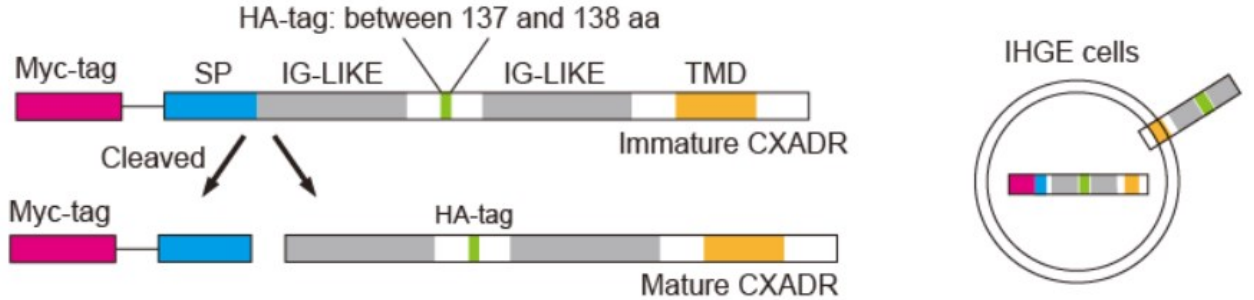

b
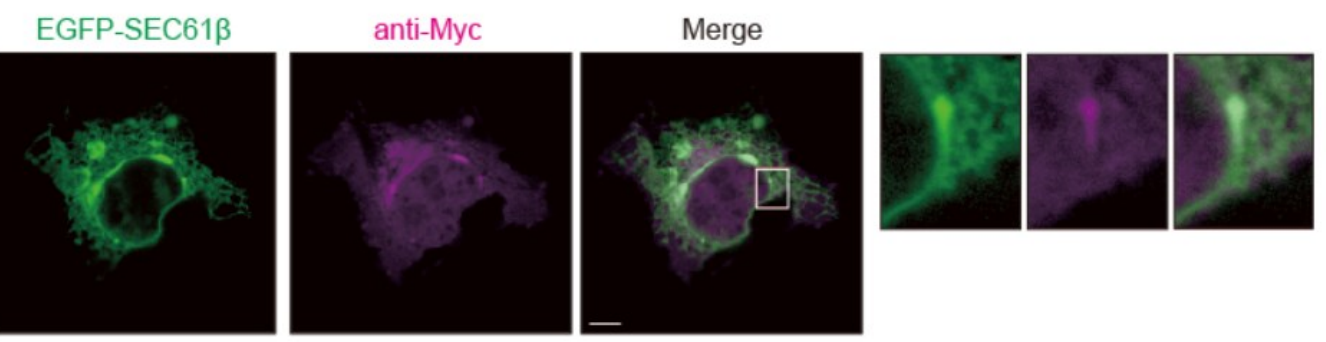

641
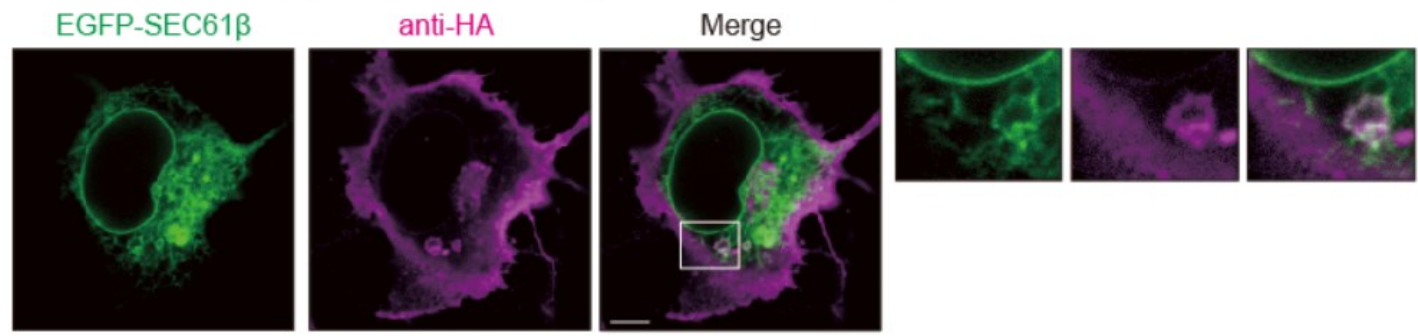

C

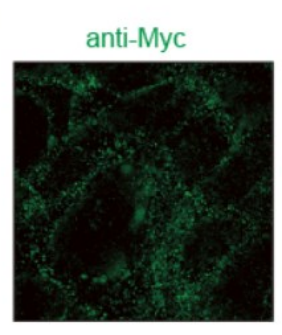

Phalloidin-
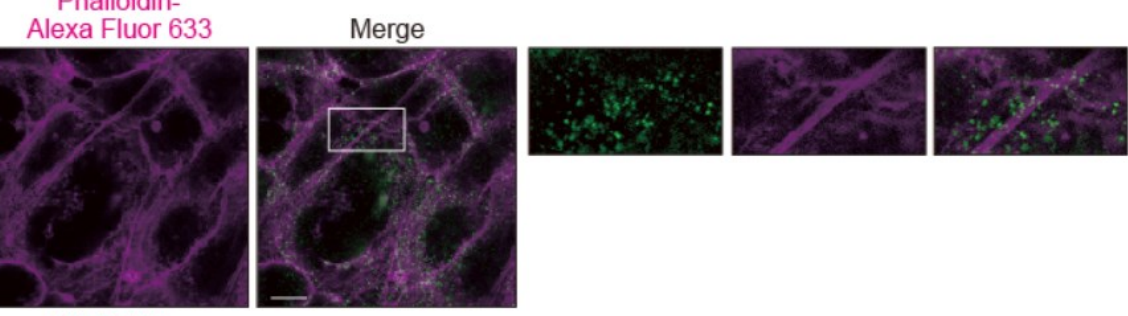

anti-HA

Phalloidin
Alexa Fluor
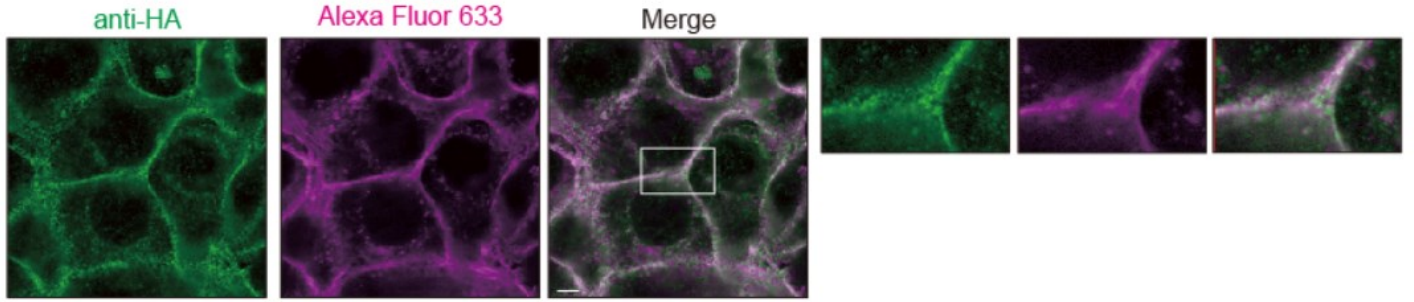

643

\section{Figure 4. Localization of CXADR in IHGE cells.}

645 (a) Schematic view of the structure of Myc-tagged HA-inserted CXADR. Immature

646 form of Myc-tagged HA-inserted CXADR was used as an internal control to monitor

647 degradation flux of mature form of HA-inserted CXADR. The Myc tag is indicated by 
648 the magenta box, and the HA-tag is indicated by the green box. The signal peptide is 649 indicated by the blue box, and IG-LIKE or TMD domains are indicated by gray boxes or 650 an orange box, respectively. (b,c) IHGE cells were transiently transfected with plasmids 651 encoding Myc-tagged HA-inserted CXADR; in (b), the cells were also transfected with 652 EGFP-SEC61 $\beta$ (green). Following $48 \mathrm{~h}$ of incubation, cells were fixed, stained with 653 anti-Myc (b, magenta; c, green) or anti-HA (b, magenta; c, green): in (c), the cells were 654 also stained with Alexa Fluor 633-conjugated phalloidin (magenta). The cells were 655 then analyzed by immunofluorescence microscopy. Higher magnification of the areas 656 indicated by white boxes in the upper panels are shown in the right side. Scale bars, 5 $657 \mu \mathrm{m}$.

658

659 
a

660

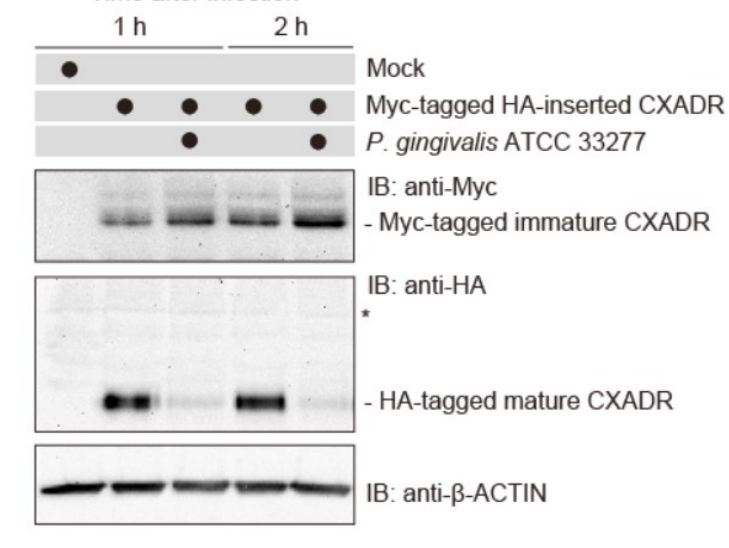

c

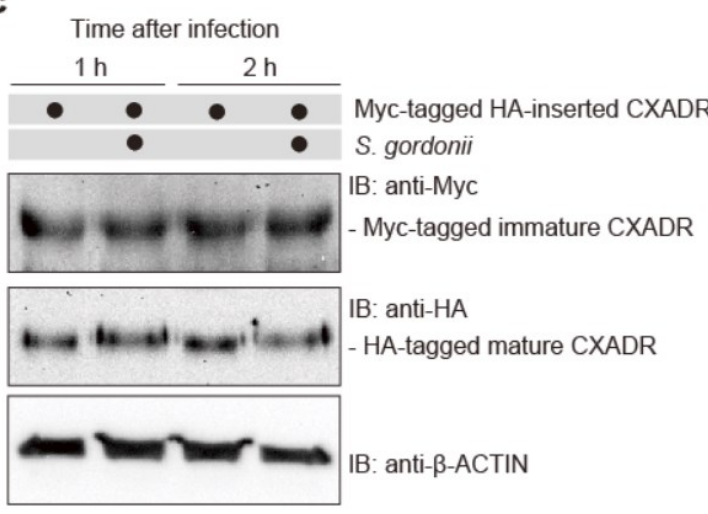

661 b Time after infection

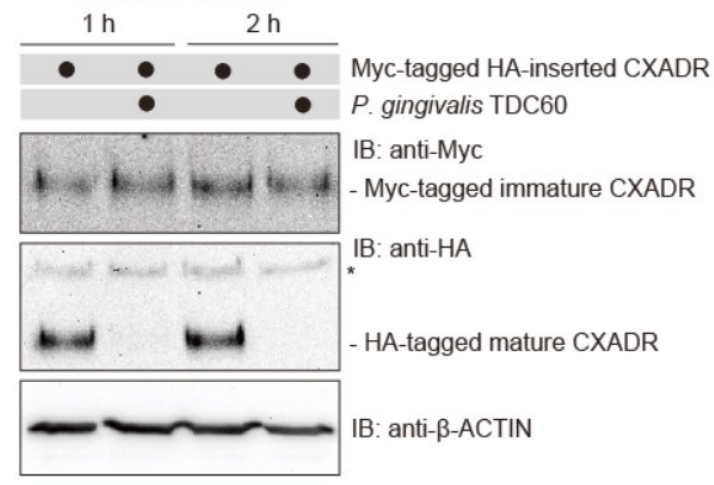

d

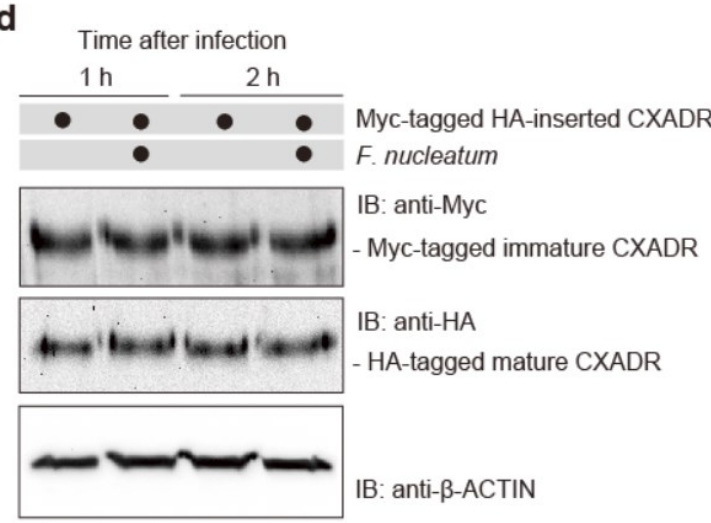

662

663 Figure 5. P. gingivalis, but not $\boldsymbol{S}$. gordonii or $\boldsymbol{F}$. nucleatum, degrades CXADR in

664 IHGE cells.

665

(a-d) IHGE cells were transiently transfected with Myc-tagged HA-inserted CXADR

plasmid. Following $48 \mathrm{~h}$ of incubation, the cells were infected with $P$. gingivalis ATCC

667

33277 (a), P. gingivalis TDC60 (b), S. gordonii DL-1 (c), or F. nucleatum ATCC 25586

668

(d) at an MOI of 100 for the indicated times. The cells were then analyzed by immunoblotting with the indicated antibodies. 
a

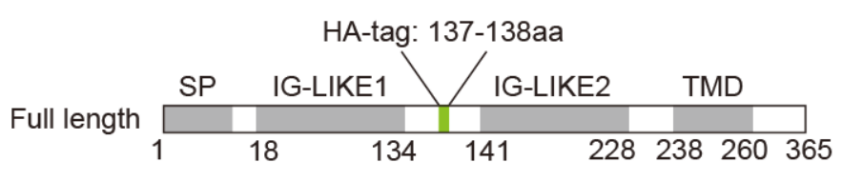

$\Delta(1-234)$

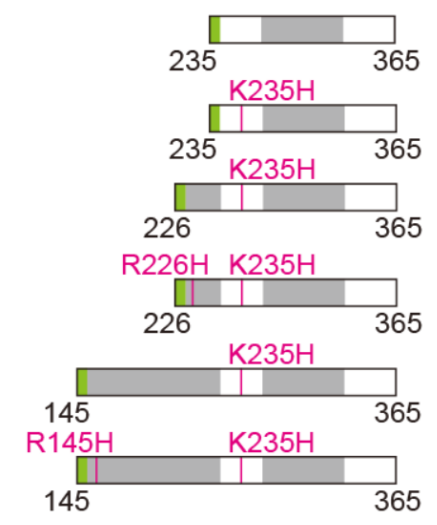

673

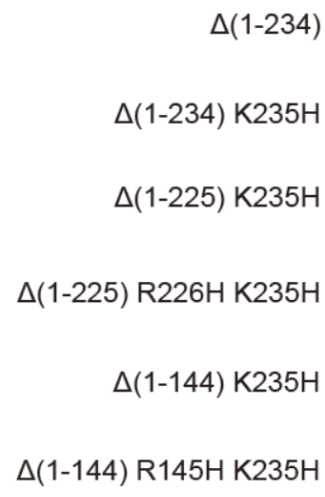

b
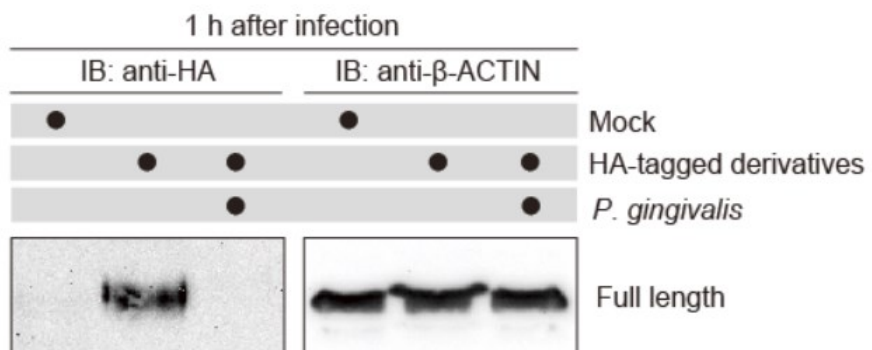

P. gingivalis

Full length
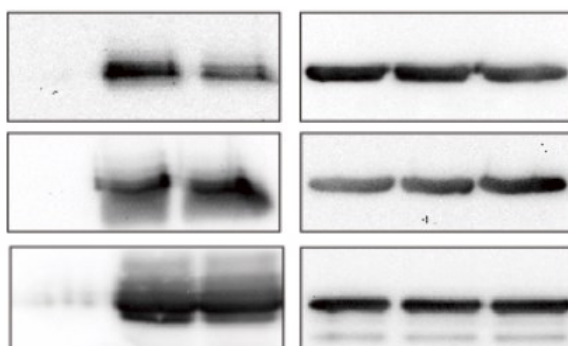

$\Delta(1-234)$

$\Delta(1-234) \mathrm{K} 235 \mathrm{H}$

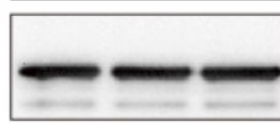

$\Delta(1-225) \mathrm{K} 235 \mathrm{H}$
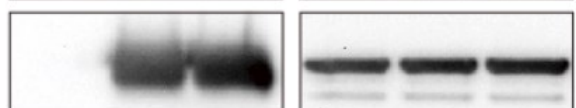

$\Delta(1-225) \mathrm{R} 226 \mathrm{H} \mathrm{K} 235 \mathrm{H}$
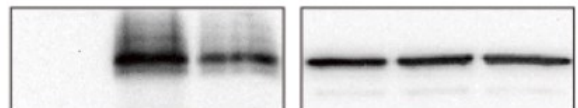

$\Delta(1-144) \mathrm{K} 235 \mathrm{H}$
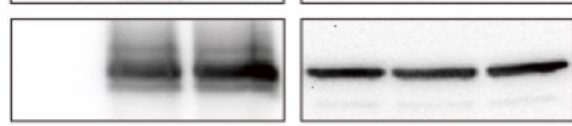

$\Delta(1-144) \mathrm{R} 145 \mathrm{H} \mathrm{K} 235 \mathrm{H}$

674

675

676 Figure 6. The R145 and K235 residues are targeted for degradation of CXADR by

$677 \boldsymbol{P}$. gingivalis in IHGE cells

678

(a) Schematic view of the CXADR structure and derivatives. SP, IG-LIKE, or TMD 
679 domains are indicated by gray boxes. HA-tag is shown in green. The point mutations $680 \mathrm{R} 145 \mathrm{H}$ and $\mathrm{K} 235 \mathrm{H}$ are shown in magenta. (b) IHGE cells were transiently transfected 681 with plasmid encoding HA-inserted CXADR or the indicated CXADR mutants. 682 Following $48 \mathrm{~h}$ of incubation, the cells were infected with $P$. gingivalis at an MOI of 100 683 for $1 \mathrm{~h}$, and then analyzed by immunoblotting using the indicated antibodies.

684

685

686 
a

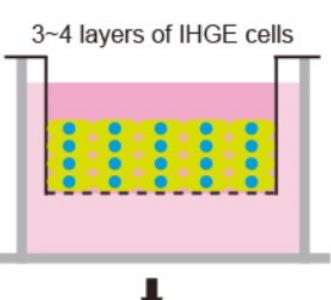

30 min of tracer administration
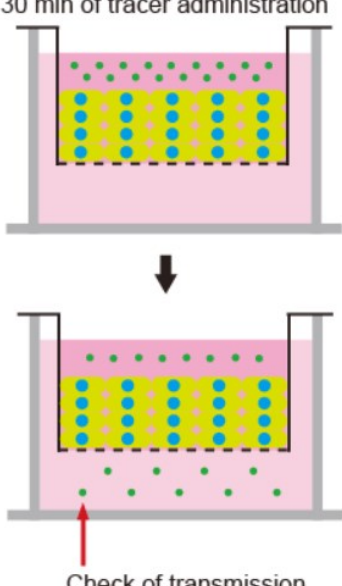

Check of transmission

687

688

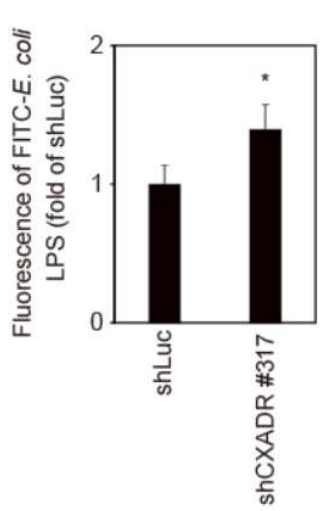

b
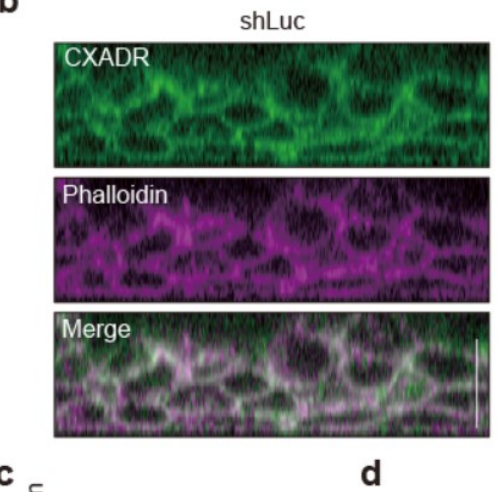

d
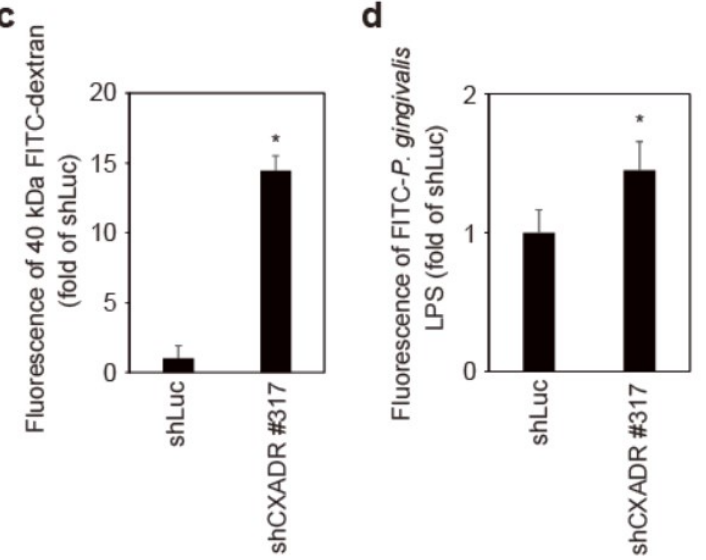

e

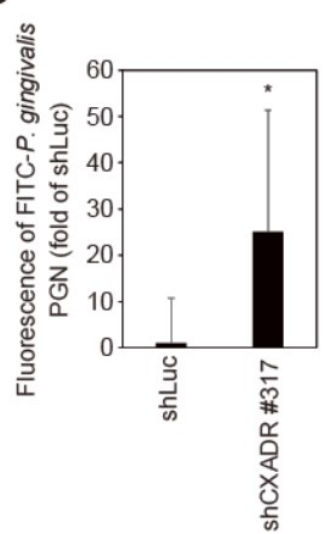

g

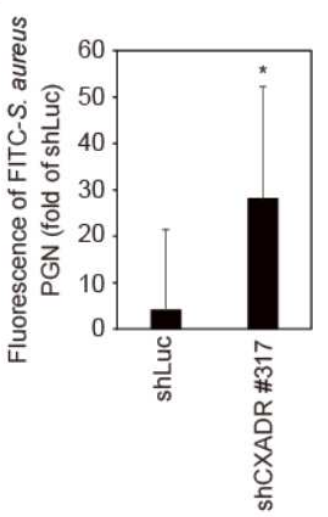

689

690 Figure 7. CXADR is required for epithelial barrier function of gingival epithelial

691 tissues.

692 (a,b) Schematic illustration (a) and confocal microscopic cross-sectional images (b) of

693 3D-tissue model expressing shLuc or shCXADR. Gingival epithelial tissues were fixed, 
694 stained with anti-CXADR (green) and Alexa Fluor 568-conjugated phalloidin (magenta), 695 and analyzed by confocal microscopy. Scale bars, $30 \mu \mathrm{m}$. (c-g) Permeability to 40 696 kDa FITC-dextran (c), FITC-P. gingivalis LPS (d), FITC-P. gingivalis PGN (e), FITC697 E. coli LPS (f), and FITC-S. aureus PGN (g) in gingival epithelial tissues expressing 698 shLuc and shCXADR. Results are expressed as fold change relative to epithelium 699 expressing shLuc and are the means \pm SD of seven technical replicates. $\quad *, p<0.05$, one700 tailed $t$ test (c-e).

701 


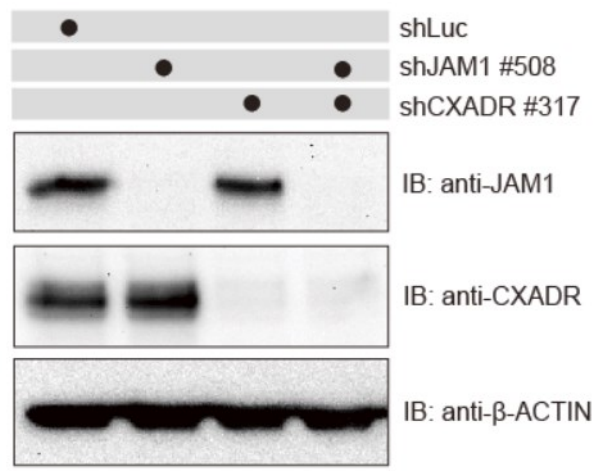

702

b
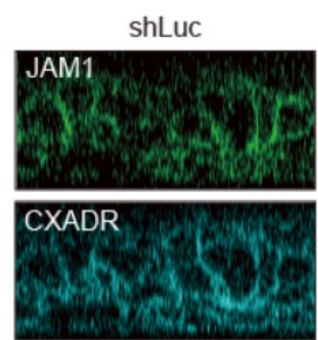

Phalloidin
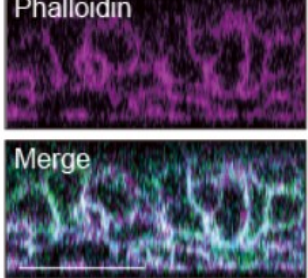

Phalloidin c

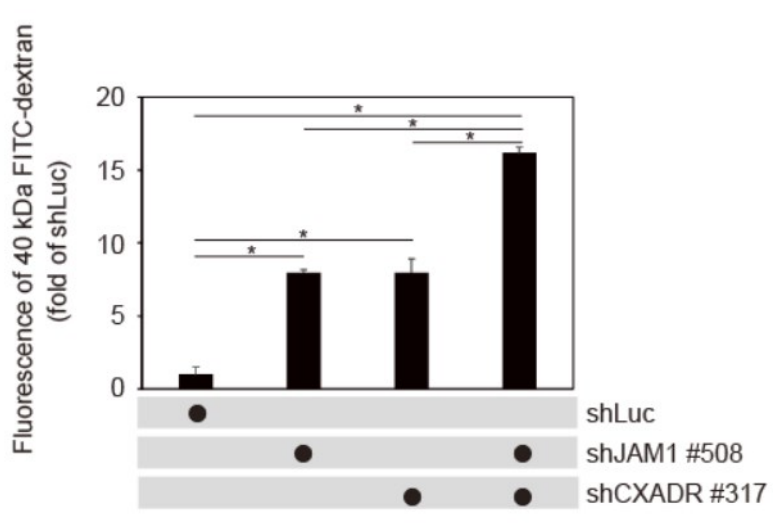

shJAM1 \#508
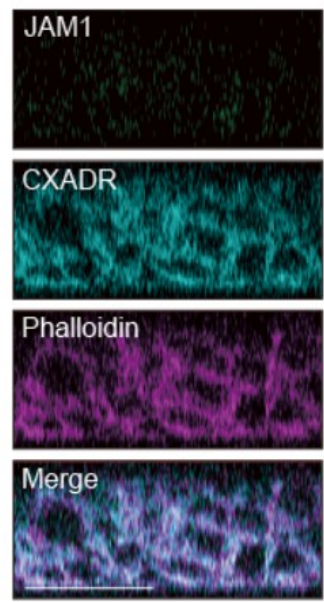

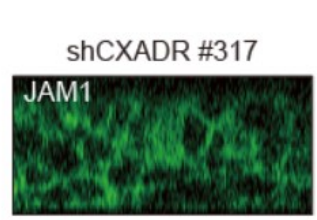

CXADR

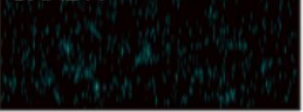

Phalloidin
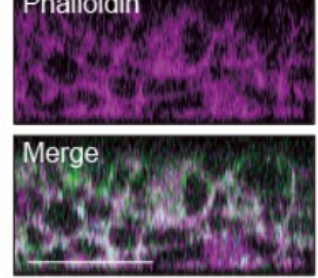

shJAM1 \#508 + shCXADR \#317

JAM1
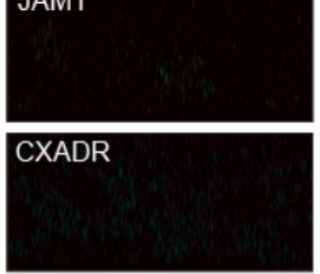

Phalloidin
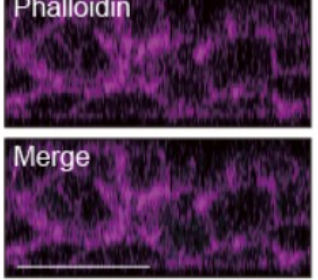

703

Figure 8. Barrier function in gingival epithelial tissues is regulated by CXADR independently of JAM1.

(a) IHGE cells stably expressing shLuc, shJAM1 \#508, shCXADR \#317, or both shJAM1 \#508 and shCXADR \#317 were analyzed by immunoblotting with the indicated

(b) Gingival epithelial tissues stably expressing shLuc, shJAM1 \#508,

710 shCXADR \#317, or both shJAM1 \#508 and shCXADR \#317 on coverslips were fixed,

711 stained with anti-JAM1 (green), anti-CXADR (cyan) and Alexa Fluor 568-conjugated

712 phalloidin (magenta), and analyzed by confocal microscopy. Scale bars, $30 \mu \mathrm{m}$.

713 Permeability to $40 \mathrm{kDa}$ FITC-dextran in gingival epithelial tissues expressing shLuc, 
714 shJAM1 \#508, shCXADR \#317, or both shJAM1 \#508 and shCXADR \#317. Results

715 are expressed as fold change relative to epithelium expressing shLuc and are the means \pm 716 SD of eight technical replicates. *, $<<0.05$, two-tailed $t$ test.

717

718 


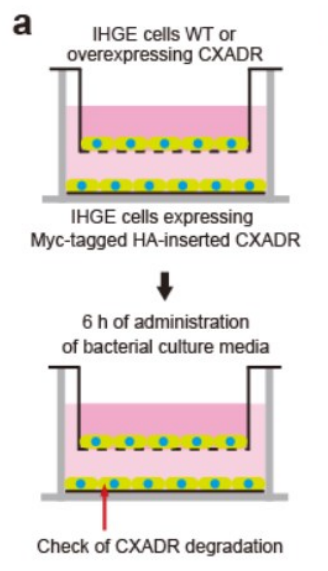

b

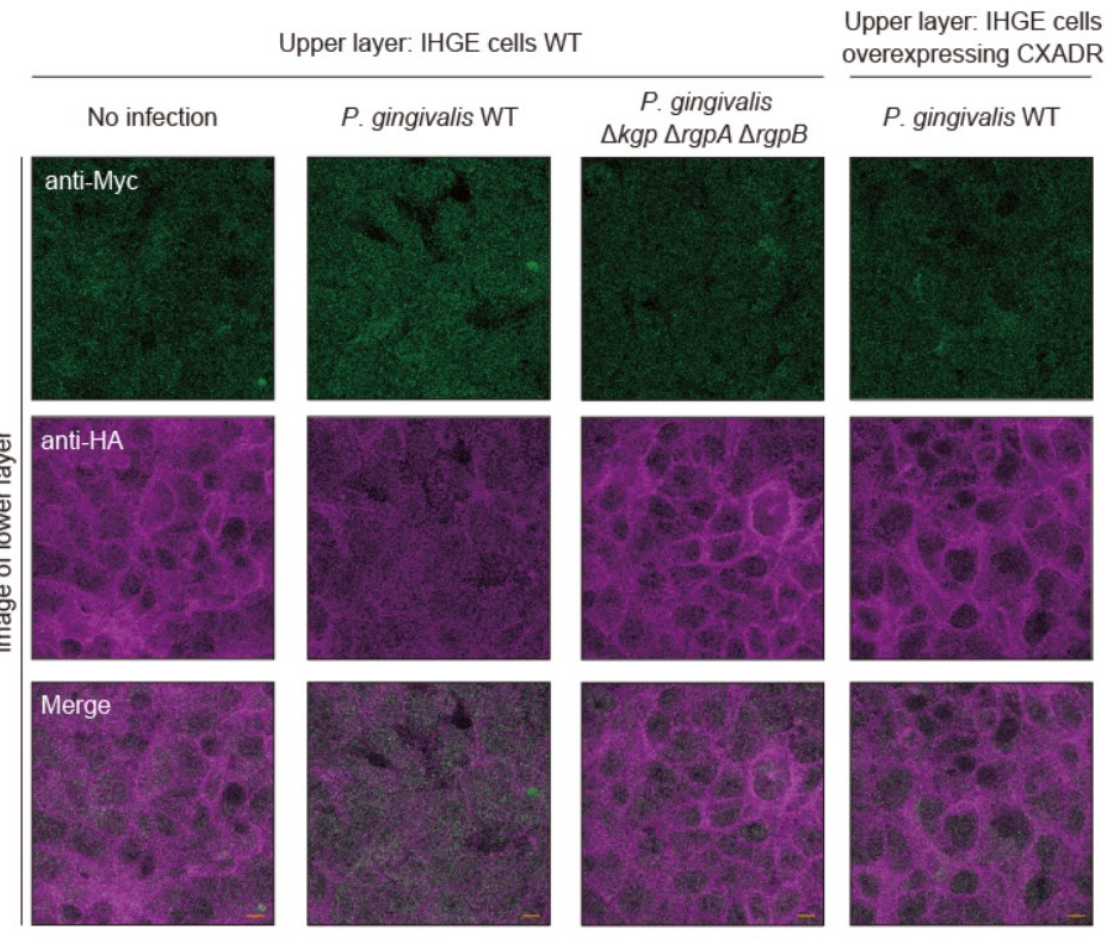

Figure 9. P. gingivalis gingipains penetrate the epithelial barrier of IHGE cells.

(a, b) Schematic image of the culture insert system (a). Gingival epithelial cells WT or stably expressing Myc-tagged HA-inserted CXADR were cultured in the upper compartment and IHGE cells stably expressing Myc-tagged HA-inserted CXADR on a coverslip in the lower compartment. The bacterial culture supernatant of $P$. gingivalis WT or or $\triangle \operatorname{kgp} \Delta \operatorname{rgp} A \Delta \operatorname{rgpB}$ mutant was administered to cells. Following $6 \mathrm{~h}$ of incubation, cells in the lower compartment were fixed, stained with anti-Myc (green), and anti-HA (magenta), and analyzed by confocal microscopy (b). Scale bars, $10 \mu \mathrm{m}$. 
a

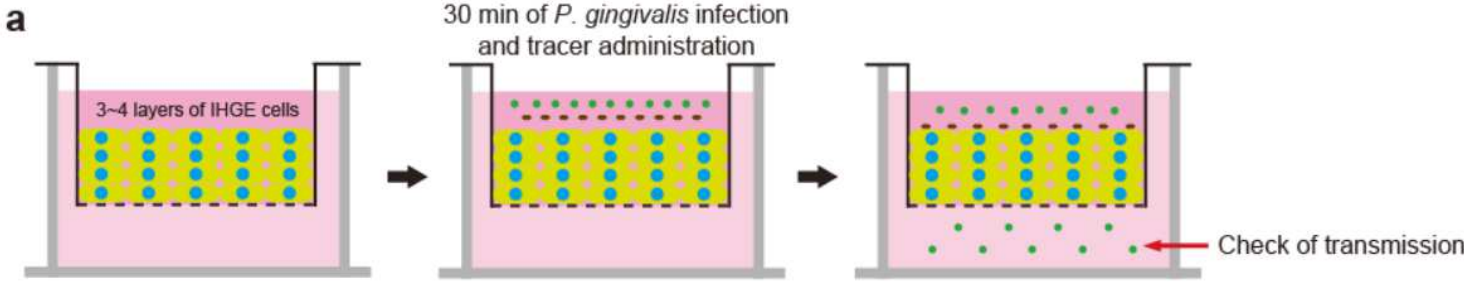

b
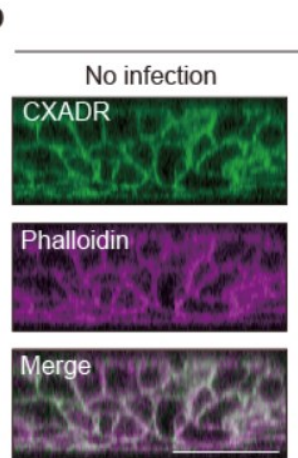

d

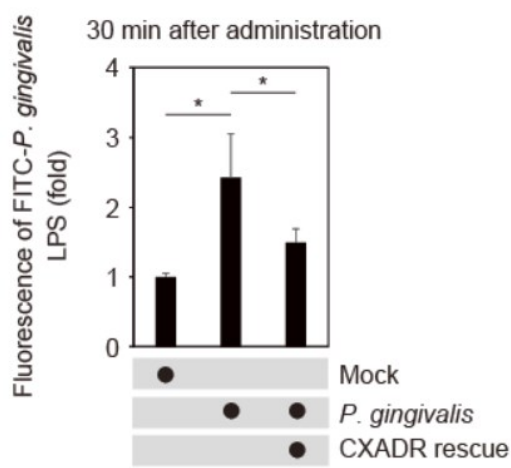

30 min after infection
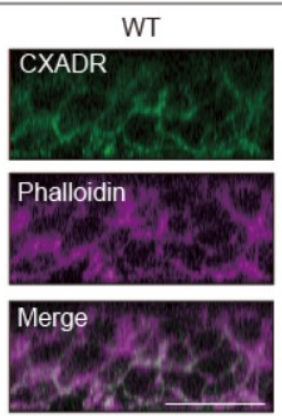

e c

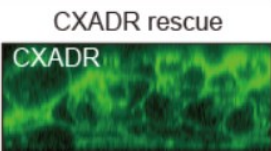

Phalloidin

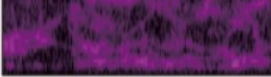

Merge

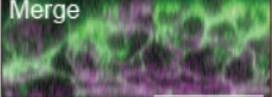

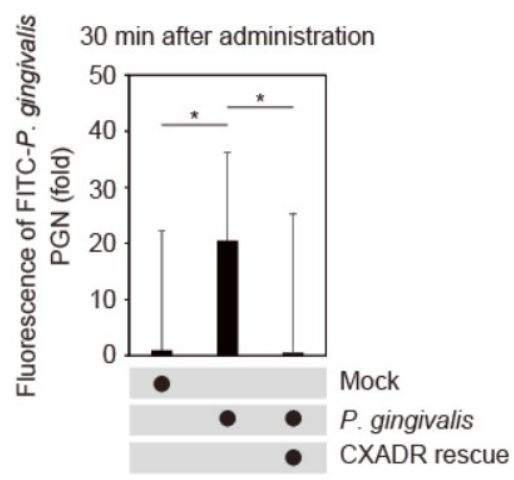

f

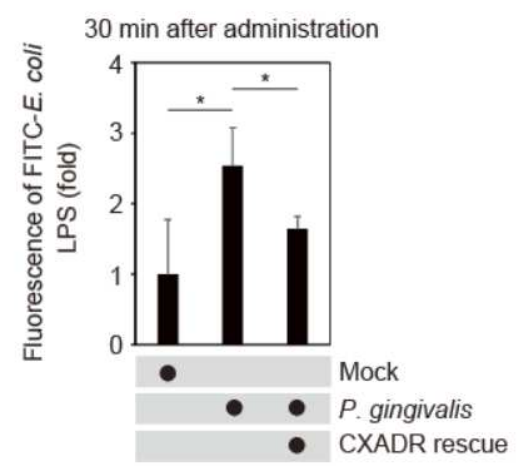

g

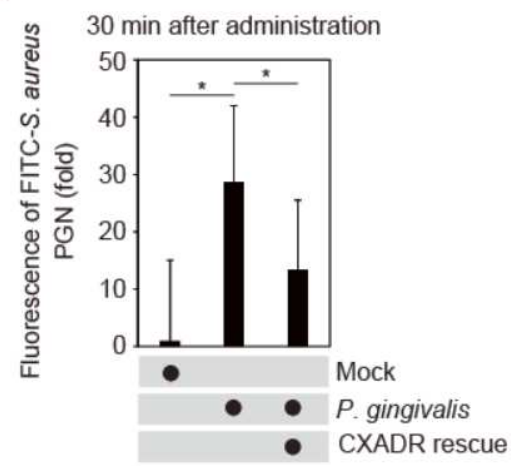

Figure 10. P. gingivalis degrades CXADR of gingival epithelium, causing penetration of LPS and PGN.

(a,b) Schematic illustration of the three-dimensional culture (a) and confocal microscopic 
$740 \mu \mathrm{m} . \quad$ (c-g) Permeability to $40 \mathrm{kDa}$ FITC-dextran (c), FITC-P. gingivalis LPS (d), FITC741 P. gingivalis PGN (e), FITC-E. coli LPS (f), and FITC-S. aureus PGN (g) of gingival 742 epithelial tissues (WT or overexpressing CXADR) infected with $P$. gingivalis. Three743 dimensional tissues on culture inserts were infected with $P$. gingivalis and FITC-labeled 744 tracer in the upper compartment. Following 30 min of incubation, the transmission of 745 tracer from the upper compartment to the lower compartment was analyzed by 746 spectrometry. Results are expressed as fold change relative to uninfected WT cells and 747 are the means \pm SD of seven technical replicates. ${ }^{*}, \mathrm{p}<0.05$, one-tailed $t$ test (closed 748 testing procedure). 


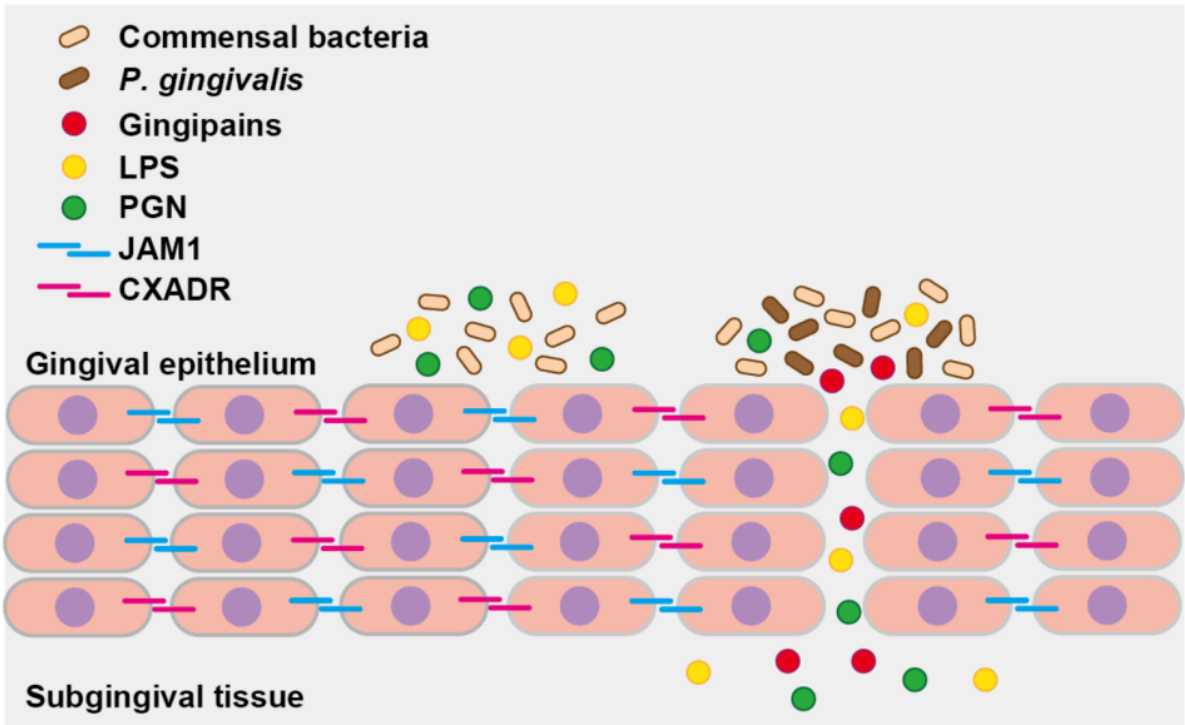

Figure 11. Proposed model of how $P$. gingivalis gingipains send bacterial virulence factors through the gingival epithelium.

In gingival epithelial tissues CXADR (magenta) and JAM1 (cyan) are not interdependent on the permeability. P. gingivalis gingipains degrade CXADR and JAM1, which increases the permeability of gingival epithelium to gingipains and other factors. Subsequently, gingipains are transferred to the deeper epithelium to further degrade CXADR and JAM1, which allows LPS and PGN to penetrate the gingival epithelium and reach subepithelial tissues. Finally, gingipains, LPS, and PGN induce inflammation in gingival tissues. 


\section{Figures}

a

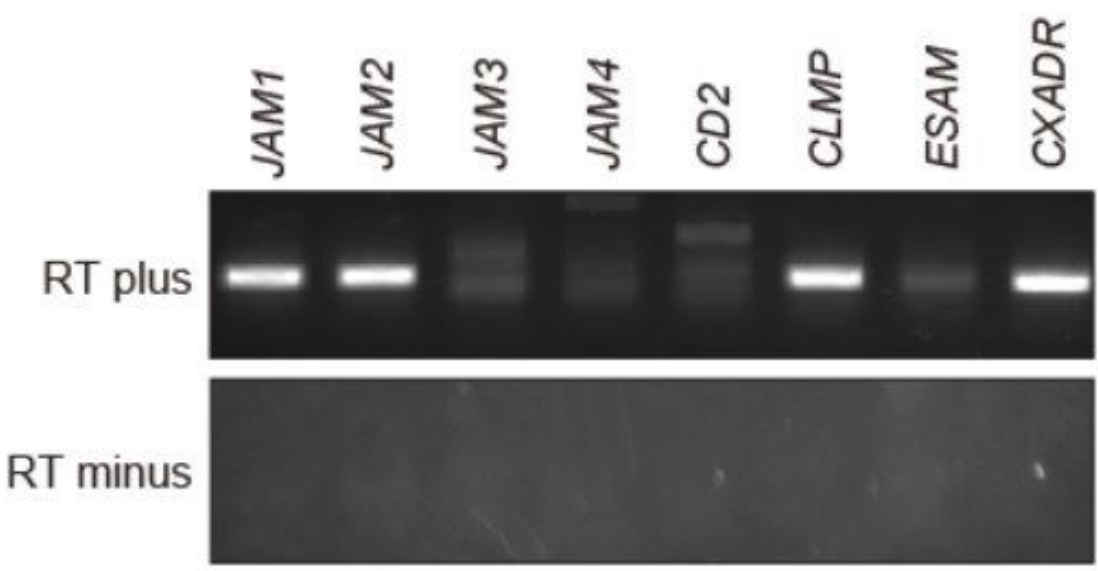

b

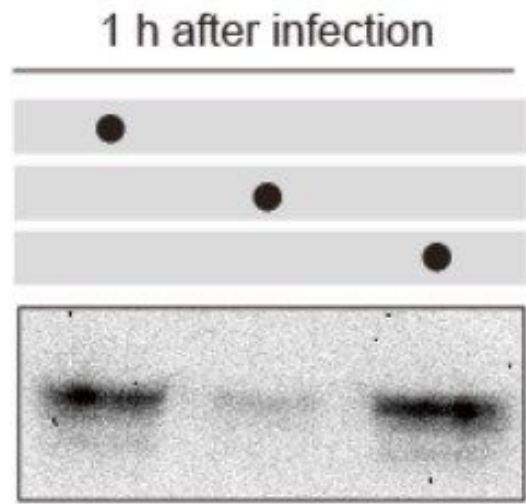

Mock

$P$. gingivalis WT

$P$. gingivalis $\triangle k g p \Delta r g p A \Delta r g p B$

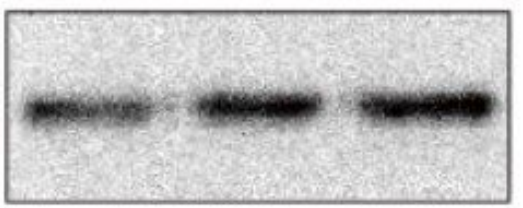

IB: anti-JAM1

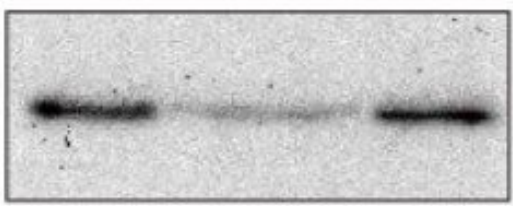

IB: anti-JAM2

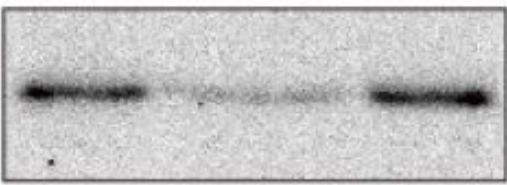

IB: anti-CXADR

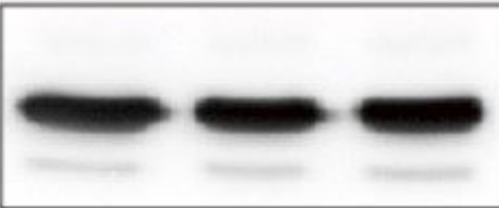

IB: anti-CLMP

IB: anti-B-ACTIN

\section{Figure 1}

P. gingivalis gingipain s degrade CXADR in IHGE cells. (aExpression of JAM1 , JAM2, JAM3 , JAM4 , CD2 , CLMP , ESAM , and CXADR by IHGE cells analyzed by RT PCR (b IHGE cells were infected with P. gingivalis WT or $\Delta \mathrm{kgp} \Delta \operatorname{rgpA} \Delta \operatorname{rgpB}$ mutant at an $\mathrm{MOI}$ of 100 for $1 \mathrm{~h}$. The cells were then analyzed by 
immunoblotting with the indicated antibodies. $\beta$ ACTIN was used as a loading control. RT, reverse transcription. IB, immunoblot.

a

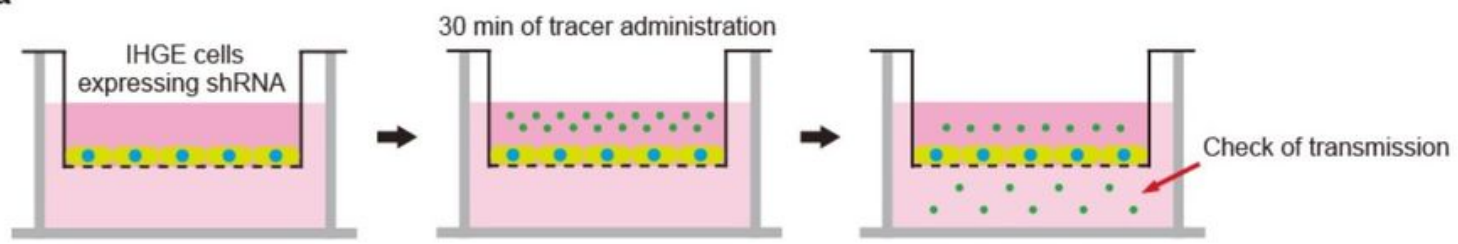

b

C
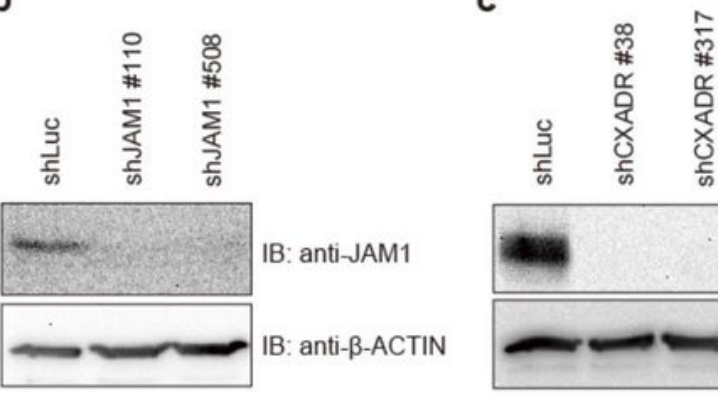

d

e
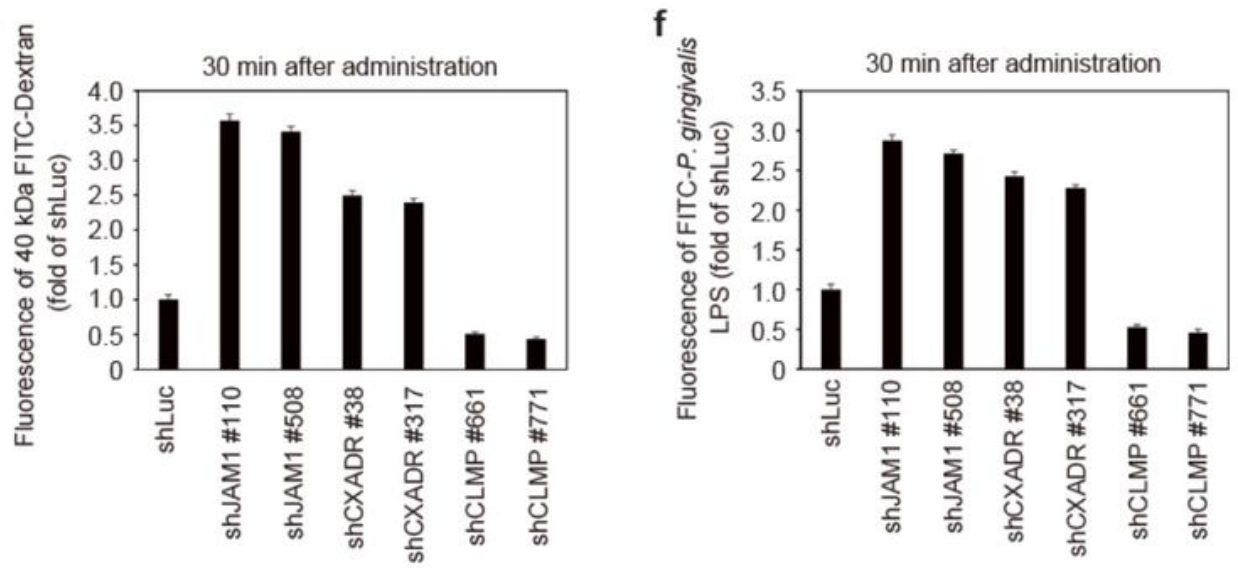

g

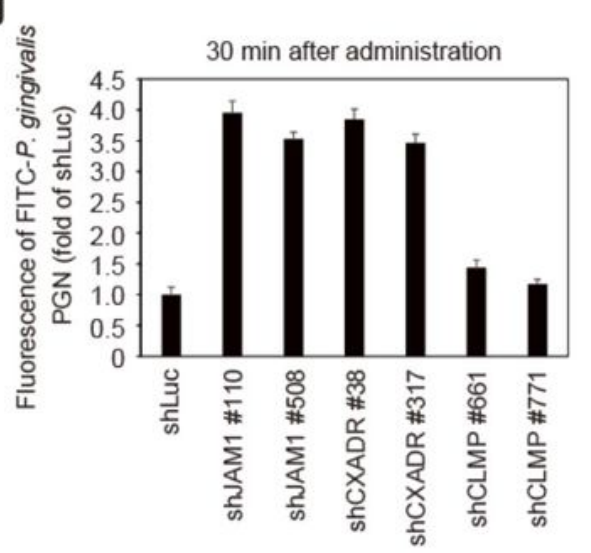

Figure 2

Loss of CXADR increased the epithelial barrier permeability. (a (a)) Schematic image of Schematic image of the culture insert system. Monolayer of IHGE cells stably the culture insert system. Monolayer of IHGE cells stably exexpressing shLuc, shJAM1 \#110, pressing shLuc, shJAM1 \#110, shJAM1 \#508shJAM1 
\#508, shCXADR \#38, shCXADR \#317, shCXADR \#38, shCXADR \#317, 613 shCLMP \#661, and shCLMP \#771 shCLMP \#661, and shCLMP \#771 were cultured in culture inserts. FITCwere cultured in culture inserts. FITC--labeled tracer labeled tracer was added to culture media in the upper cowas added to culture media in the upper compartment. Following 30 min of incubation, mpartment. Following 30 min of incubation, the transmission of tracer from the upper compartment to the lower compartment was the transmission of tracer from the upper compartment to the lower compartment was analyzed by spectrometry. analyzed by spectrometry. (b(b-dd)) IHGE cells stably exIHGE cells stably expressing pressing shLuc, shJAM1, shLuc, shJAM1, shCXADR, or shCLMPshCXADR, or shCLMP werewere analyzed by immunoblotting wanalyzed by immunoblotting with the indicated antibodies. ith the indicated antibodies. (e(e-ff)) Permeability to $40 \mathrm{kDa}$ FITCPermeability to $40 \mathrm{kDa}$ FITC-dextran (dextran (ee))," FITCFITC--P. gingivalisP. gingivalis LPS (LPS (ff), FITC), FITC--P. P. gingivalisgingivalis PGN (PGN (gg) in IHGE cells expressing indicated shRNA) in IHGE cells expressing indicated shRNA. Results are expressed. Results are expressed as fold change relative to cells expressing shLucas fold change relative to cells expressing shLuc and are the means \pm SD of eight technical and are the means \pm SD of eight technical replicates. replicates. 
a

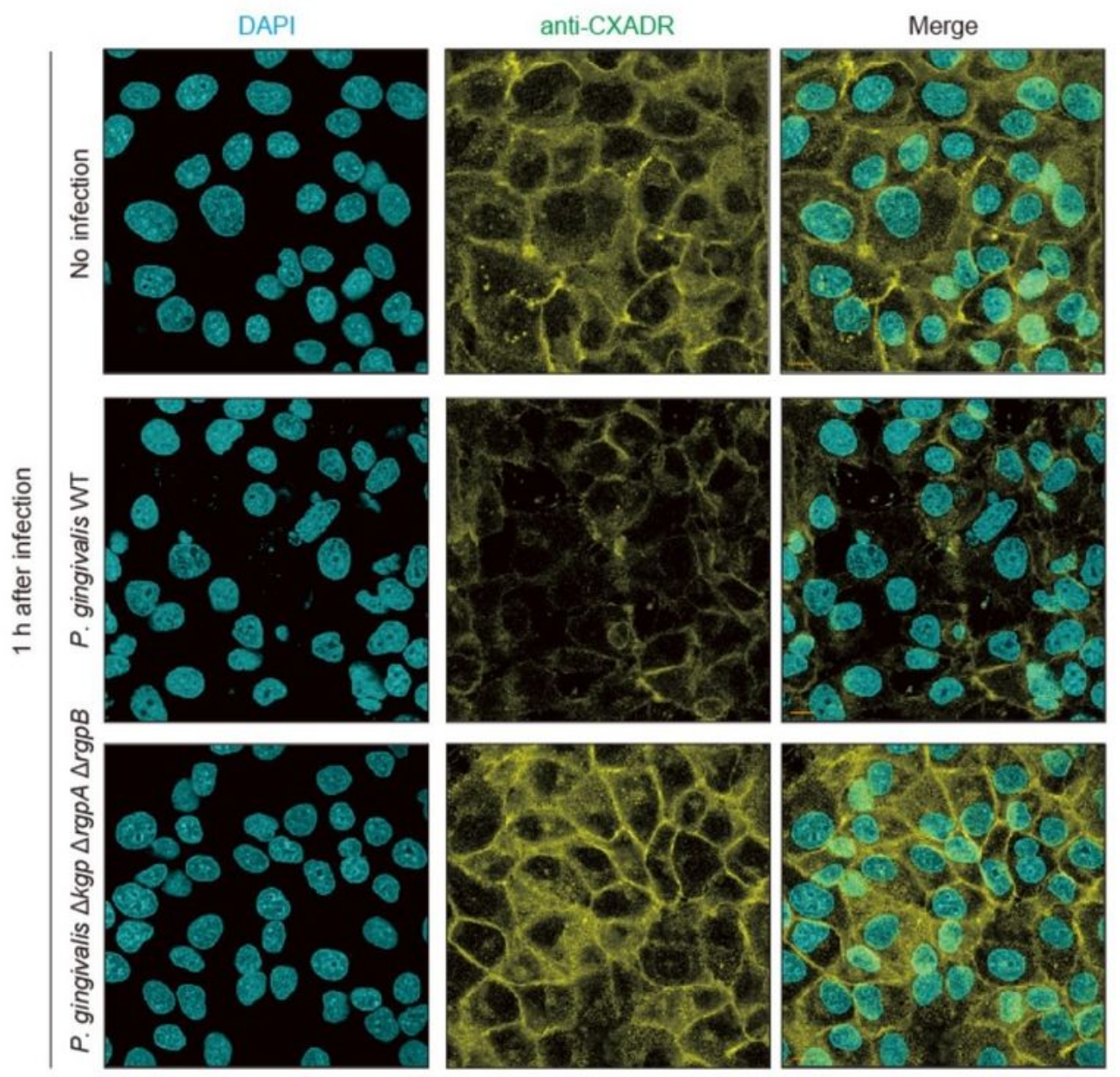

b
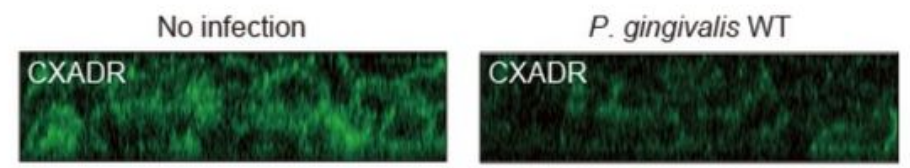

P. gingivalis $\triangle k g p \triangle r g p A \triangle r g p B$

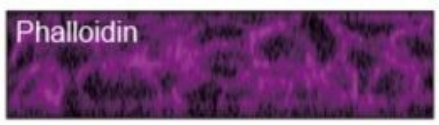

Phalloidin
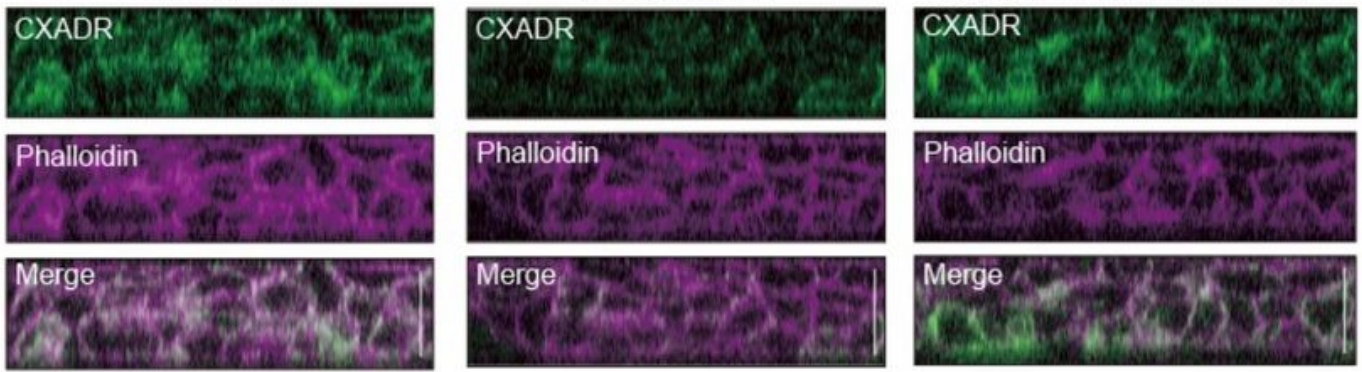

\section{Figure 3}

Degradation of CXADR in IHGE cells infected with P. gingivalis gingipains (a IHGE cells were infected with P. gingivalis WT or $\Delta \mathrm{kgp} \Delta \operatorname{rgpA} \Delta \mathrm{rgpB}$ mutant at an $\mathrm{MOI}$ of 100 for $1 \mathrm{~h}$. The cells were then fixed, staine d with DAPI (cyan) and anti CXADRCXADR (yellow), and analyzed by confocal microscopy. Scale bars, 10 $\mu \mathrm{m}$. (yellow), and analyzed by confocal microscopy. Scale bars, $10 \mu \mathrm{m}$. ((bb)) GGingival epithelial tissues on coverslips were infected with ingival epithelial tissues on coverslips were infected with P. gingivalisP. 
gingivalis WT or WT or $\Delta \Delta \mathrm{kgpkgp} \Delta \Delta \operatorname{rgpArgpA} \Delta \Delta \operatorname{rgpBrgpB}$ mutantmutant for $2 \mathrm{~h}$. for $2 \mathrm{~h}$. The tissues The tissues were thwere then fixed, stained with antien fixed, stained with anti--CXADRCXADR 6 (green(green) and Alexa Fluor 568) and Alexa Fluor 568--conjugated phconjugated phalloidin (magenta), and analyzed by confocal alloidin (magenta), and analyzed by confocal microscopy. Scale bars, $30 \mu \mathrm{m}$.microscopy. Scale bars, $30 \mu \mathrm{m}$.

a
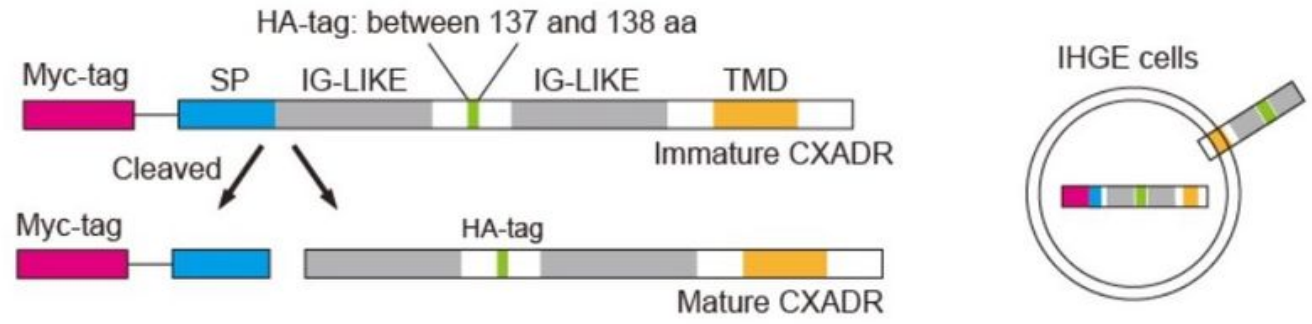

b
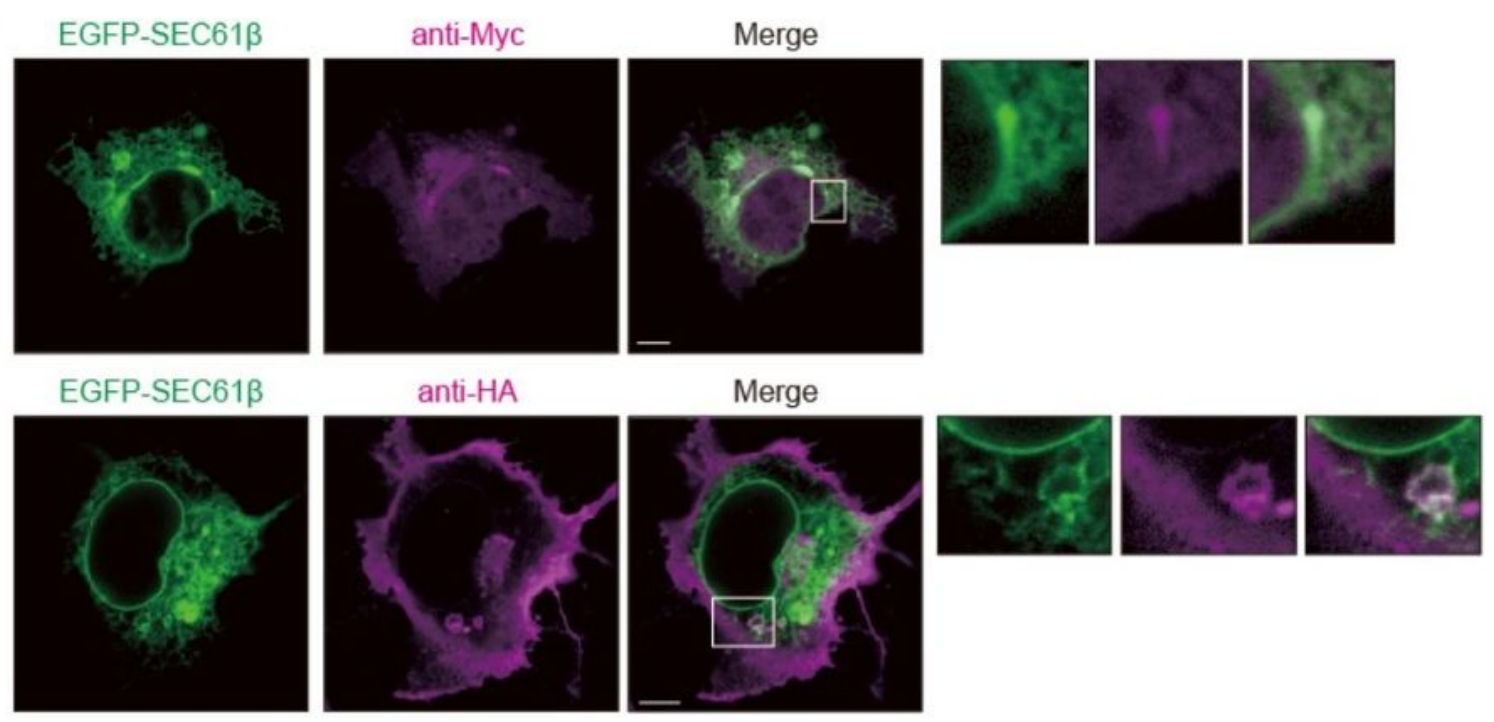

c

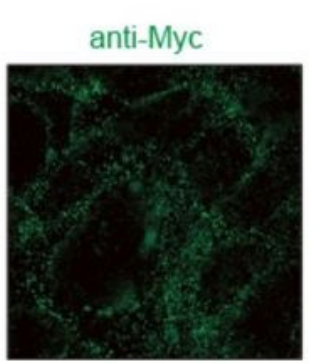

PhalloidinAlexa Fluor 633
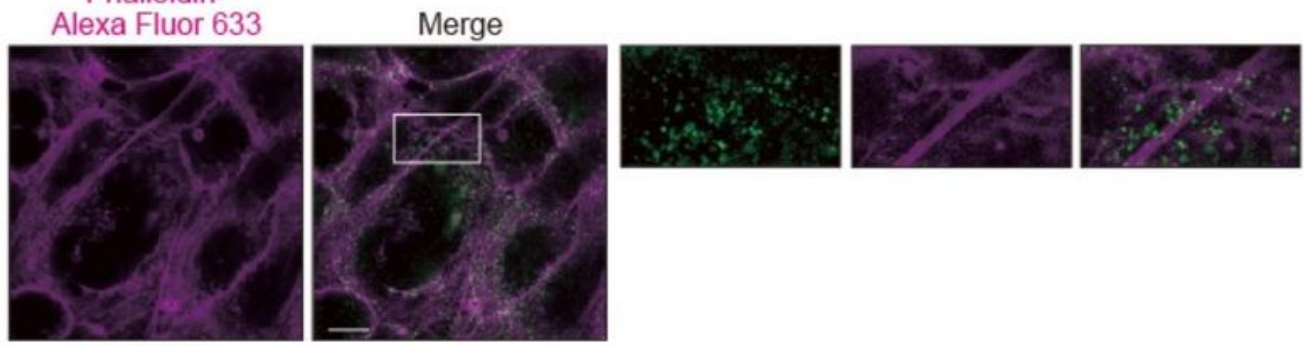

Phalloidin-
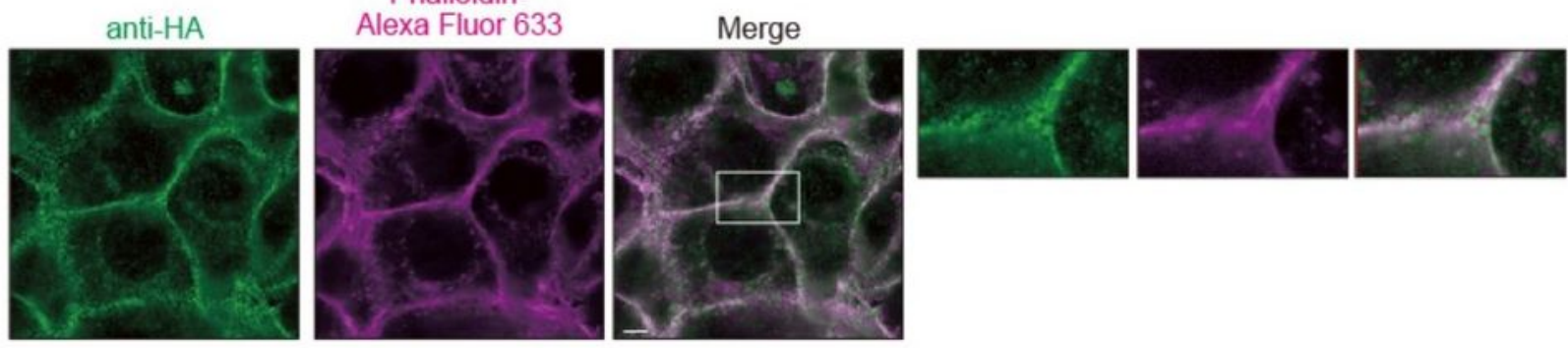

Figure 4 
Localization of CXADR in IHGE cells. (aSchematic view of the structure of Myc tagged HA inserted CXADR. Immature form of Myc tagged HA inserted CXADR was used as an internal control to monitor degradation flux of mature form of HA inserted CXADR. The Myc tag is indicated by the magenta box, athe magenta box, and the HAnd the HA-tag is indicated by the green box. tag is indicated by the green box. The signal peptide is The signal peptide is indicated by the blue box, and IGindicated by the blue box, and IG-LIKE or TMD domains are indicated by gray boxes or LIKE or TMD domains are indicated by gray boxes or an orange box, respectively. an orange box, respectively. ((bb, „cc)) IHGE cells were transiently transfected with plasmids IHGE cells were transiently transfected with plasmids encoding Mycencoding Myc--ttagged HAagged HA--inserted CXADR; in (inserted CXADR; in (bb), the cells were also

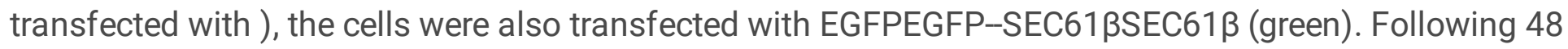
$\mathrm{h}$ of incubation, cells were(green). Following $48 \mathrm{~h}$ of incubation, cells were fixed, stained with fixed, stained withantianti-Myc (b, magenta; c, green) or antiMyc (b, magenta; $c$, green) or anti-HA (b, magenta; cHA (b, magenta; c, green), green): in (c: in (c)), , the cells werethe cells were also stained with Alexa Fluor 633also stained with Alexa Fluor 633--conjugated phalloidin (magenta).conjugated phalloidin (magenta). The cells were The cells were 654 then analyzed by immunofluorescence microscopy.then analyzed by immunofluorescence microscopy. Higher magnification of the areas Higher magnification of the areas indicated by white boxes in the upper panels are shown in the right side.indicated by white boxes in the upper panels are shown in the right side. Scale bars, 5 Scale bars, $5 \mu \mathrm{m} . \mu \mathrm{m}$. 
a
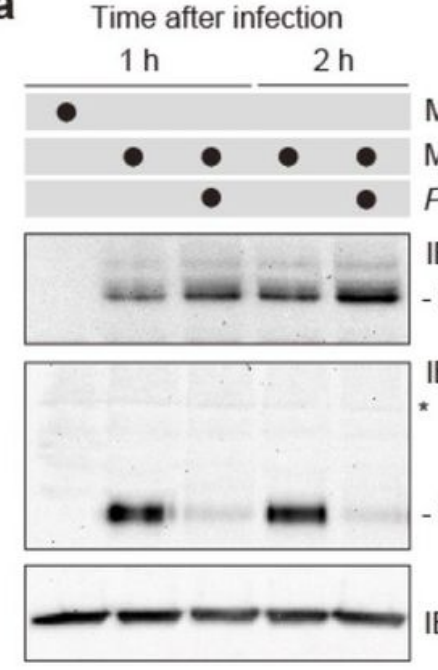

IB: anti-HA

- HA-tagged mature CXADR

IB: anti- $\beta-A C T I N$

c

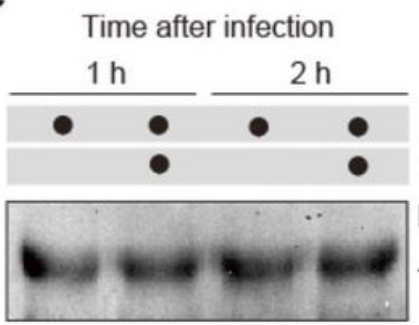

Myc-tagged HA-inserted CXADR

S. gordonii

IB: anti-Myc

- Myc-tagged immature CXADR

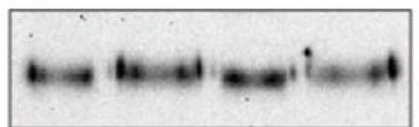

IB: anti-HA

- HA-tagged mature CXADR

IB: anti-B-ACTIN b

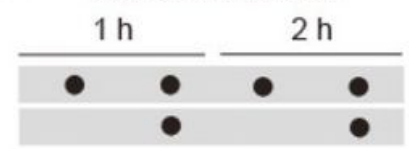

Myc-tagged HA-inserted CXADR

$P$. gingivalis TDC60

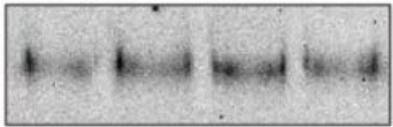

IB: anti-Myc

- Myc-tagged immature CXADR

IB: anti-HA

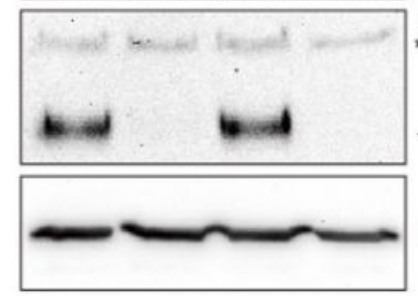

- HA-tagged mature CXADR

IB: anti- $\beta-A C T I N$

d

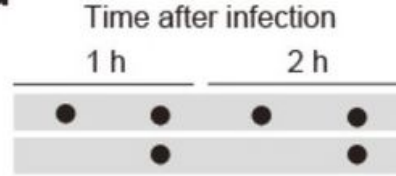

Myc-tagged HA-inserted CXADR

F. nucleatum

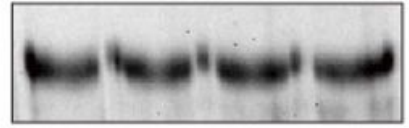

IB: anti-Myc

- Myc-tagged immature CXADR

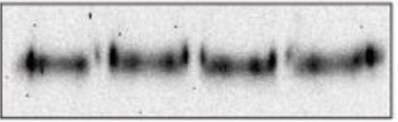

IB: anti-HA

- HA-tagged mature CXADR

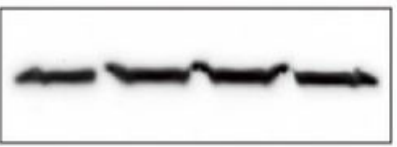

IB: anti- $\beta-A C T I N$

\section{Figure 5}

P. gingivalis, but not S. gordonii or F. nucleatum, degrades CXADR in 663 IHGE cells. (a d IHGE cells were transiently transfected with Myc tagged HA inserted CXADR plasmid. Following $48 \mathrm{~h}$ of incubation, the cells were infected with P. gingivalis ATCC 33277 (a ), P. gingivalis TDC60 (b ), S. gordonii DL 1 c ), or F. nucleatum ATCC $25586 \mathrm{~d}$ ) at an $\mathrm{MOI}$ of 100 for the indicated times. The cells were then analyzed by immunoblotting with the indicated antibodies. 
a

HA-tag: 137-138aa
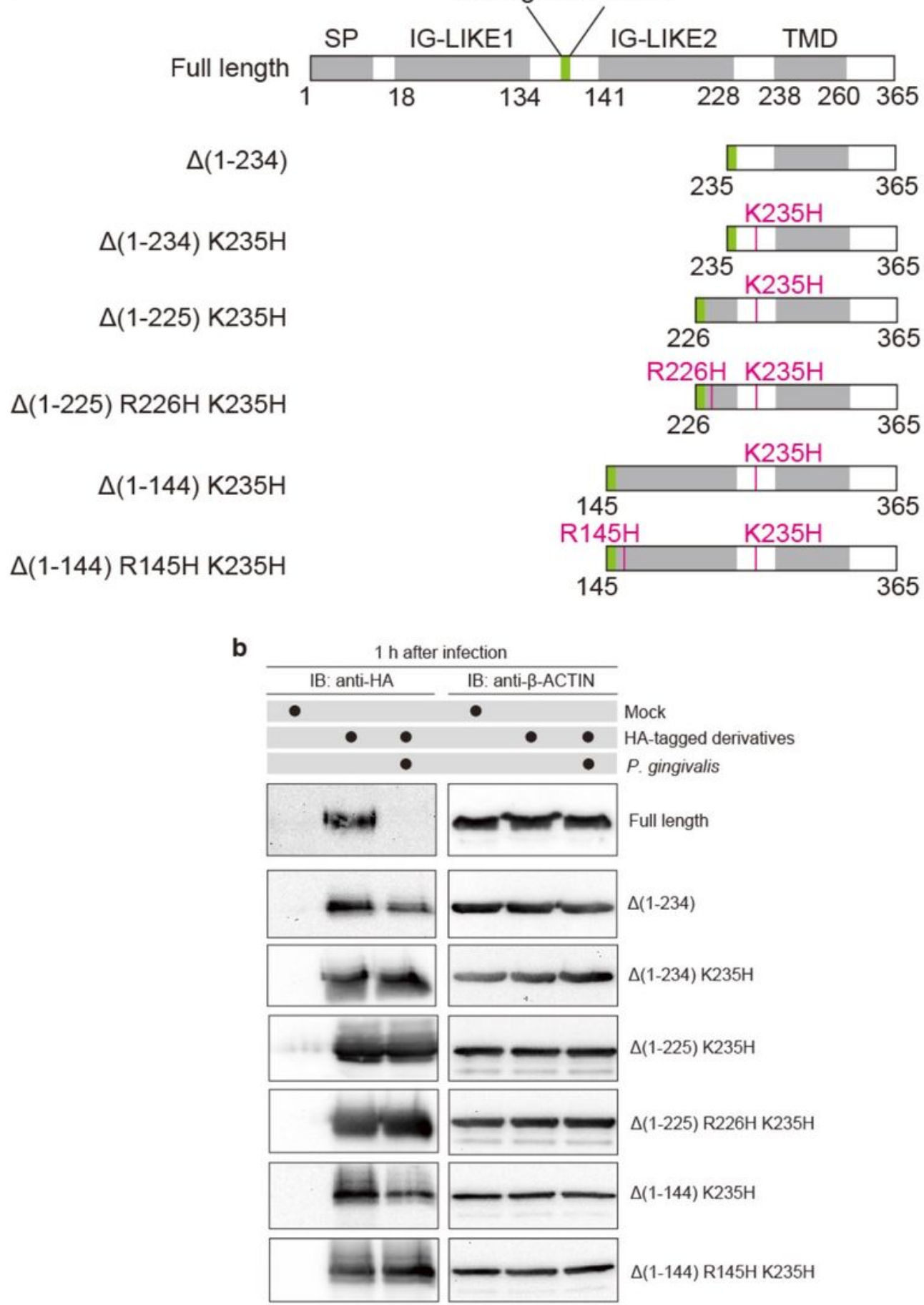

\section{Figure 6}

The R145 and K235 residues are targeted for degradation of CXADR by P. gingivalis in IHGE cells (a Schematic view of the CXADR structure and derivatives. SP, IG LIKE, or TMD domains aredomains are indicated byindicated by gray boxes. HAgray boxes. HA-tag tag is is shown in green. The point mutationshown in green. The point mutationss R145HR145H and and $\mathrm{K} 235 \mathrm{HK} 235 \mathrm{H}$ are shown in magenta. are shown in magenta. $(b(b))$ IHGE cells were transiently transfected IHGE cells were transiently 
transfected with plasmid with plasmid encoding encoding HAHA--inserted CXADR or the indicated CXADRinserted CXADR or the indicated CXADR mutantmutantss. . Following $48 \mathrm{~h}$ of incubation, Following $48 \mathrm{~h}$ of incubation, the the cells were infected with cells were infected with P. gingivalisP. gingivalis at an $\mathrm{MOI}$ of 100 at an $\mathrm{MOI}$ of 100 for $1 \mathrm{hfor} 1 \mathrm{~h}$, , andand then analyzed by immunoblotting using the indicated antibodies.then analyzed by immunoblotting using the indicated antibodies.

a

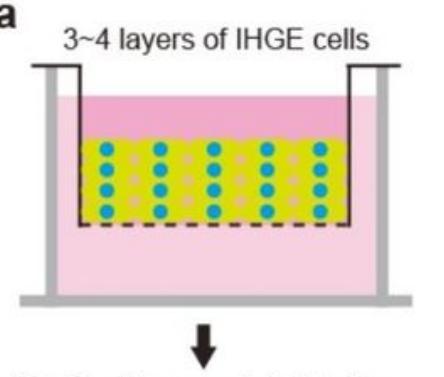

30 min of tracer administration

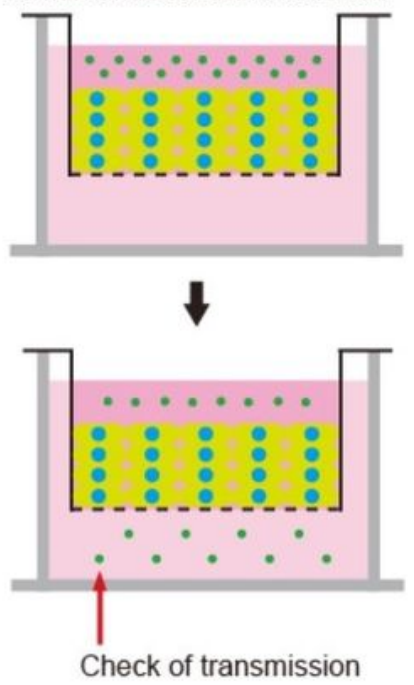

b
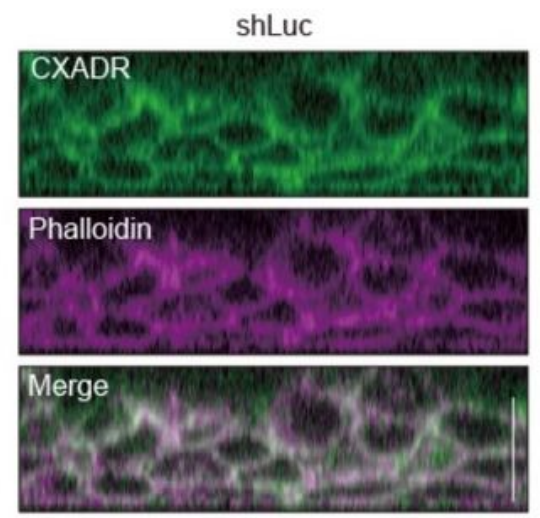

C

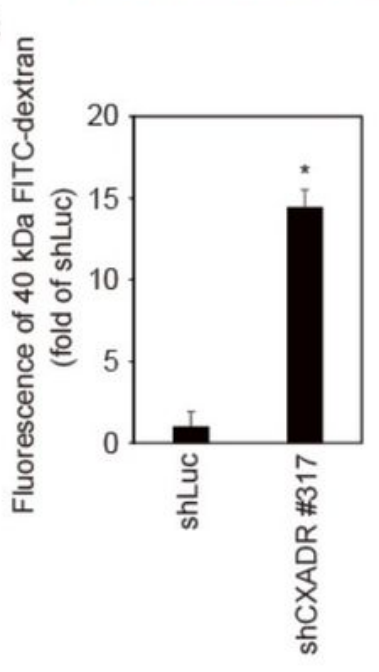

d

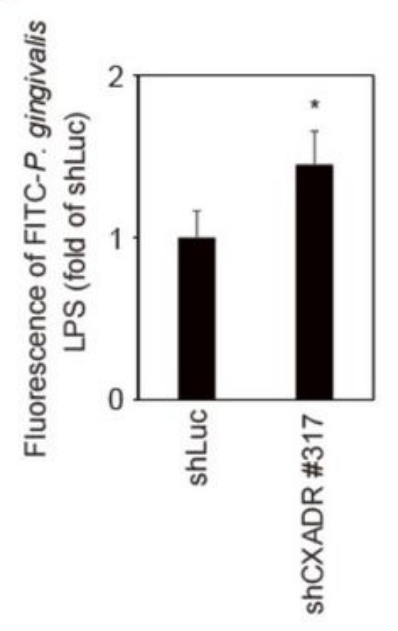

shCXADR \#317
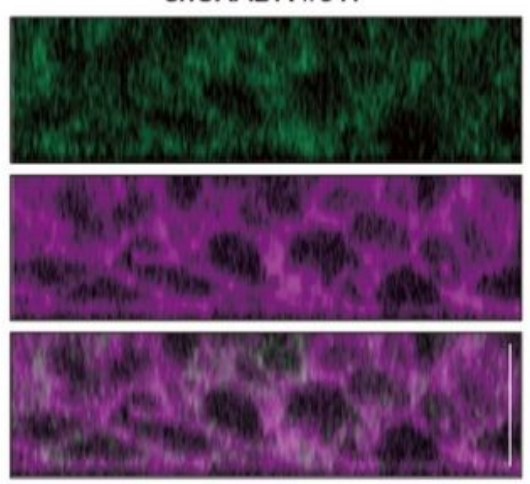

e

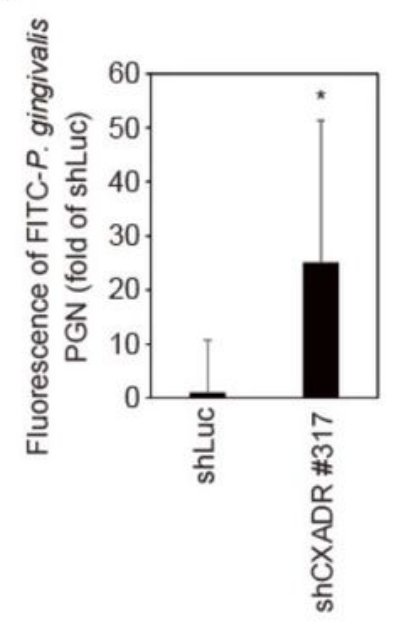

f

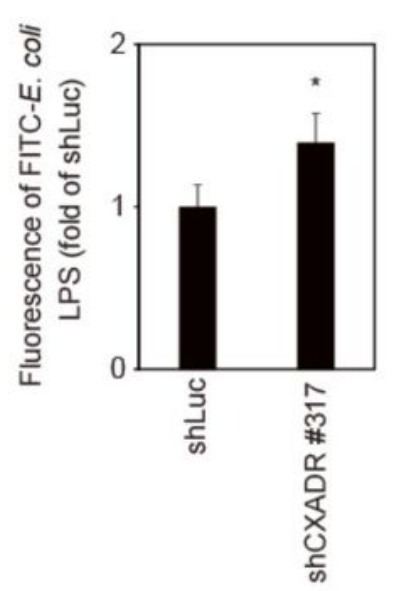

g

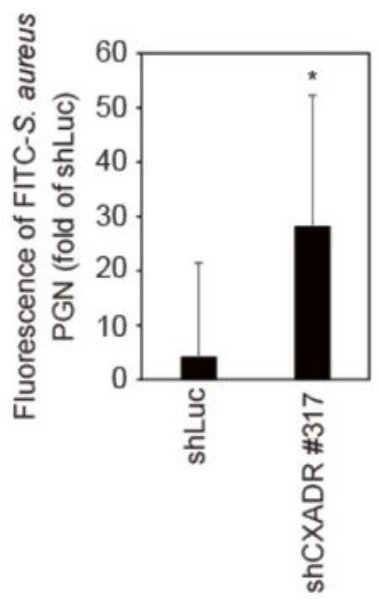


CXADRis required for epithelial barrier function of gingival epithelial tissues. ( $a, b$ Schematic illustration ( a ) and confocal microscopic cross sectional images (b) of 3D tissue model expressing shLuc or shCXADR . Gingival epithelial tissues were fixed, stained with anti stained with anti-CXADRCXADR (green(green) and Alexa Fluor 568) and Alexa Fluor 568--conjugated pconjugated phalloidin (magenta), halloidin (magenta), and analyzed by confocal microscopy. Scale bars, $30 \mu \mathrm{m}$.and analyzed by confocal microscopy. Scale bars, $30 \mu \mathrm{m}$. (c(c--gg)) Permeability to 40 Permeability to $40 \mathrm{kDa}$ FITCkDa FITC-dextran (dextran (cc), FITC), FITC--P. gingivalisP. gingivalis LPS (LPS (dd), FITC), FITC--P. gingivalisP. gingivalis PGN (PGN (ee), FITC), FITC--E. coli E. coli LPS (LPS (ff), and FITC), and FITC--S. aureus S. aureus PGN (PGN (gg) in gingival epithelial tis) in gingival epithelial tissues expressing sues expressing shLuc and shCXADRshLuc and shCXADR. Results are expressed as fold. Results are expressed as fold change relative to change relative to epithelium epithelium expressing shLuc and are the meanexpressing shLuc and are the meanss \pm SD of \pm SD of seven seven technical replicates. ${ }^{*}, p<0.05$, onetechnical replicates. *, $p<0.05$, one-- tailed tailed tt test (test (cc--ee).).

a

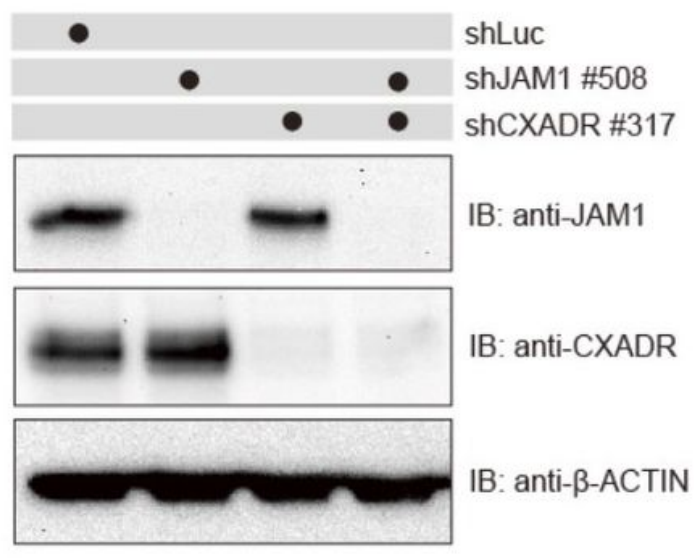

b
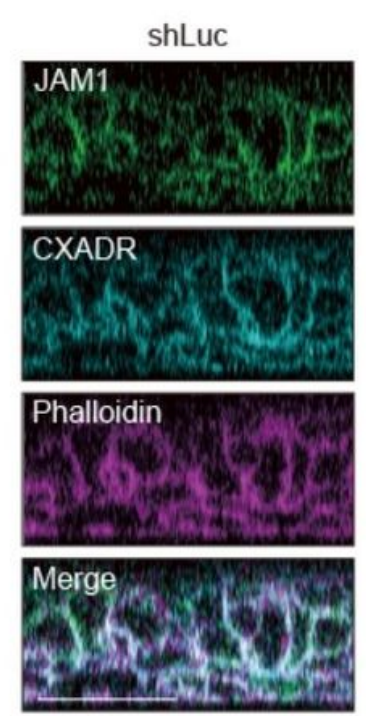

Phalloidin
C

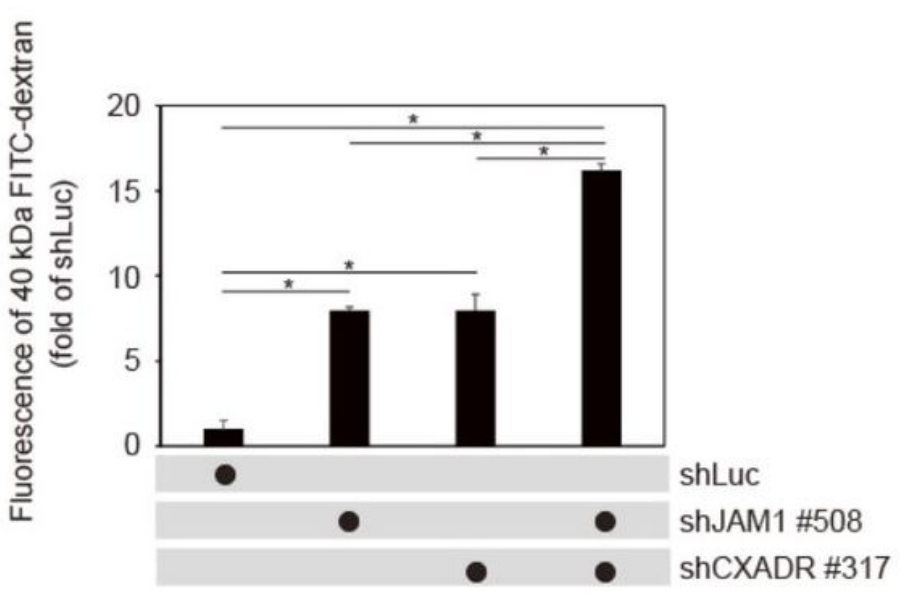

ShJAM1 \#508
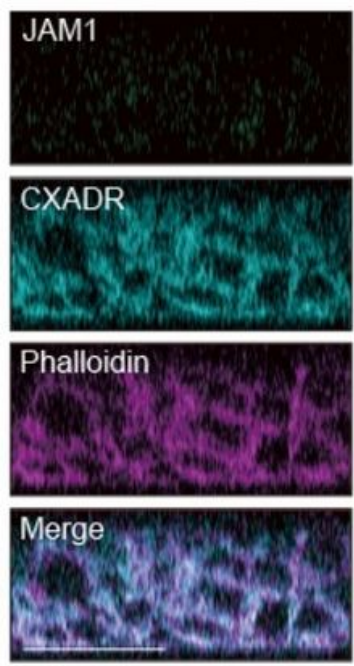

ShCXADR \#317
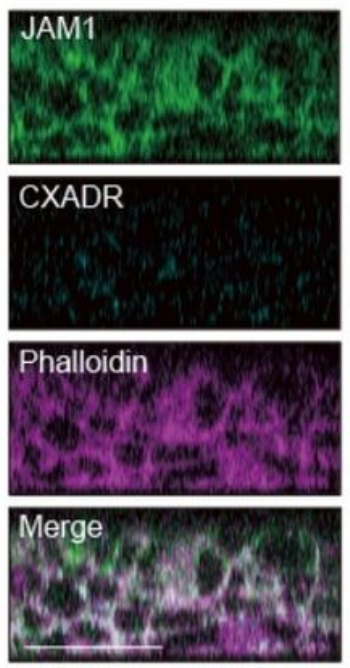

Phalloidin
ShJAM1 \#508
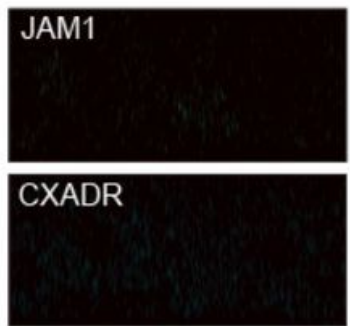

Phalloidin

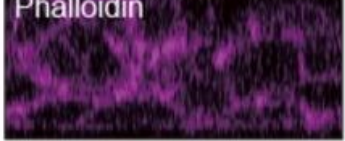

Merge
+ shCXADR \#317

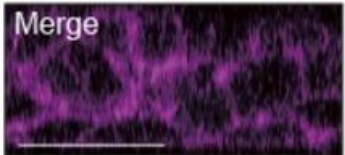




\section{Figure 8}

Barrier function in gingival epithelial tissues is regulated by CXADR 705 independently of JAM1. (a IHGE cells stably ex pressing shLuc, shJAM1 \#508, shCXADR \#317, or both shJAM1 707 \#508 and shCXADR \#317 were analyzed by immunoblotting with the indicated antibodies. (b Gingival epithelial tissues stably expressing shLuc, shJAM1 \#508, shCXADR \#317, or both shJAM1 \#508 and shCXADR \#317 on coverslips were fixed, stained with anti JAM1 (green), anti CXADR (cy an) and Alexa Fluor 568 conjugated phalloidin (magenta), and analyzed by confocal microscopy. Scale bars, $30 \mu \mathrm{m}$. (c Permeability to $40 \mathrm{kDa}$ FITC dextran in gingival epithelial tissues expressing shLuc, shJAM1 shJAM1 \#508\#508, shCXADR, shCXADR \#317\#317, or both shJAM1, or both shJAM1 \#508\#508 and shCXADRand shCXADR \#317\#317. Results . Results are expressed as fold change relative to epithelium expressing shLuc and are the means \pm are expressed as fold change relative to epithelium expressing shLuc and are the means \pm SD of eight technical replicatSD of eight technical replicates. ${ }^{*}, p<0.05$, twoes. ${ }^{*}, p<0.05$, two--tailed tailed tt test.test.

a

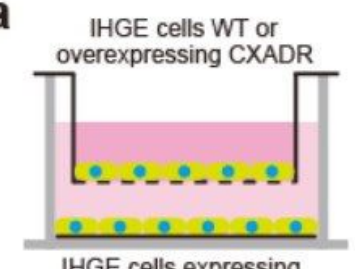

IHGE cells expressing Myc-tagged HA-inserted CXADR

\section{$\downarrow$}

$6 \mathrm{~h}$ of administration of bacterial culture media

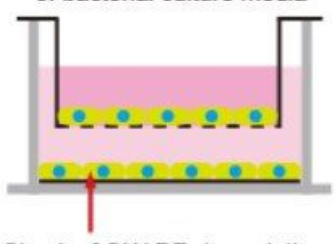

Check of CXADR degradation b

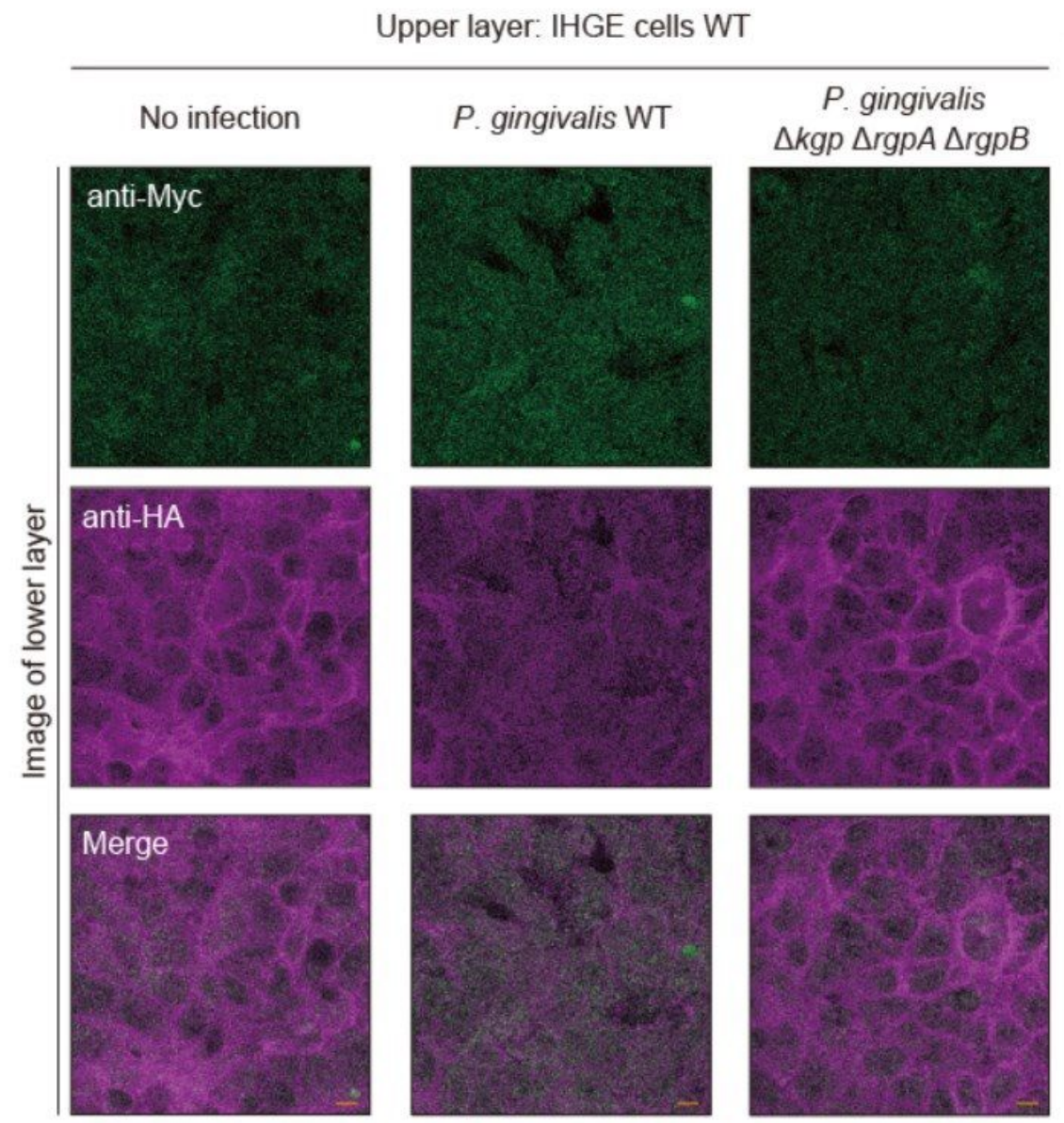

Upper layer: IHGE cells overexpressing CXADR

P. gingivalis WT
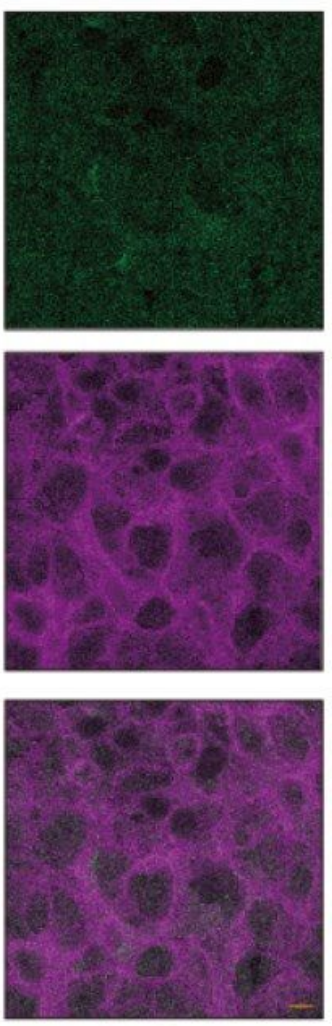

\section{Figure 9}

P. gingivalis gingipains penetrate the epithelial barrier of IHGE cells. (a, b Schematic image of the culture insert system ( a ). Gingival epithelial cell s WT or stably expressing Myc tagged HA inserted CXADR were cultured in the upper compartment and IHGE cells stably expressing Myc tagged HA inserted CXADR on a 
coverslip in the lower compartment. The bacterial culture supernatant of P. gingivalis WT or or $\Delta \mathrm{kgp} \Delta$ $\operatorname{rgpA} \Delta \mathrm{rgpB}$ mutan $\mathrm{t}$ was administered to cells. Following $6 \mathrm{~h}$ of incubation, cells in the lower compartment were fixe d, stained with anti Myc green ), and anti HA (magenta), and analyzed by confocal microscopy (b ). Scale bars, $10 \mu \mathrm{m}$.

a

$30 \mathrm{~min}$ of $P$. gingivalis infection

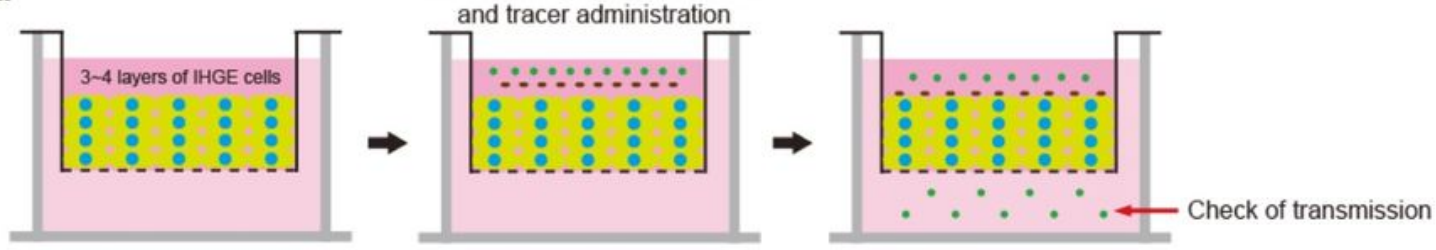

b

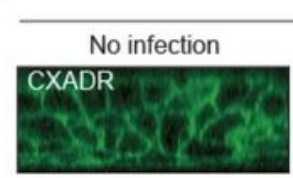

Phalloidin
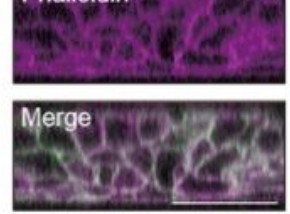

d

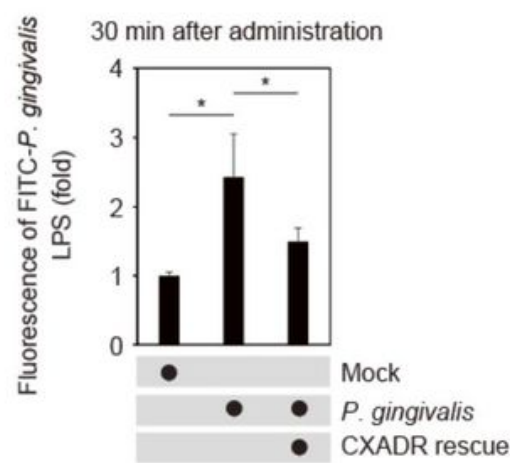

f

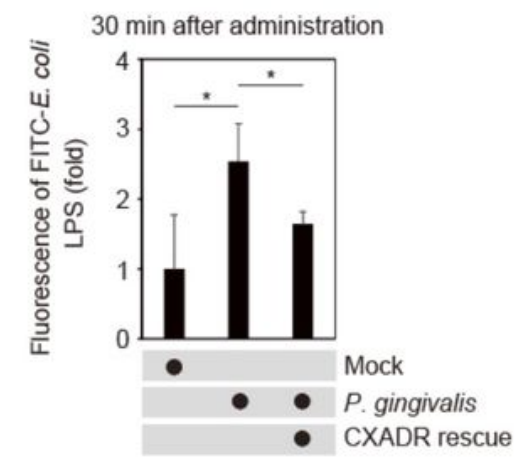

30 min after infection

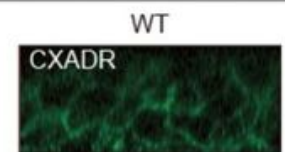

Phalloidin

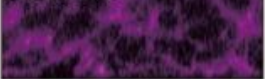

Merge

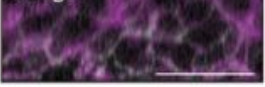

e c

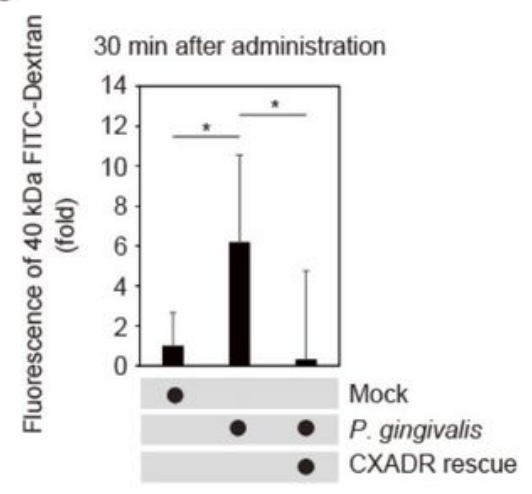

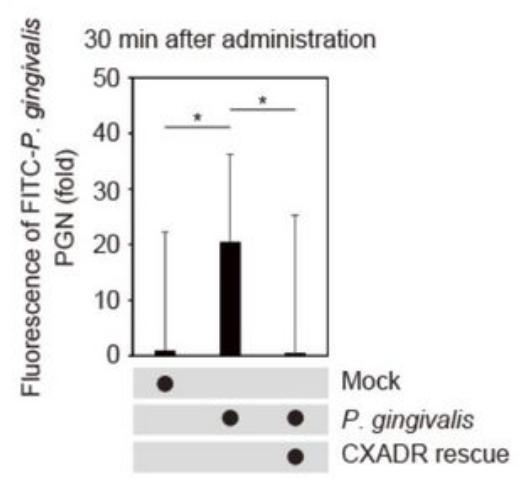

g

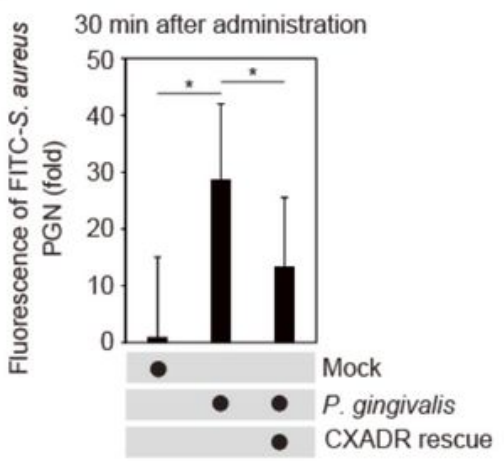

Figure 10 
P. gingivalis degrades CXADR of gingival epithelium causing penetration of LPS and PGN. (a,bSchematic illustration of the three dimensional culture ( $\mathrm{a}$ ) and confocal microscopic cross cross-sectional images (sectional images (bb) of the three) of the three--dimensional culture of IHGE cells. Gingival dimensional culture of IHGE cells. Gingival epithelial tissues epithelial tissues ((WT or overexpressing CXADRWT or overexpressing CXADR)) were infected with were infected with P. gingivalisP. gingivalis for 30 for 30 min. Tissuemin. Tissues were ths were then fixed, stained with antien fixed, stained with anti-CXADR (greenCXADR (green) and Alexa Fluor 568) and Alexa Fluor 568--738 conjugatedconjugated pphalloidin (mahalloidin (magenta), and analyzed by confocal microscopy. Scale bars, 30 genta), and analyzed by confocal microscopy. Scale bars, $30 \mu \mathrm{m} . \mu \mathrm{m}$. (c(c--gg)) Permeability to $40 \mathrm{kDa}$ FITCPermeability to 40 kDa FITC--dextran (dextran (cc), FITC), FITC--P. gingivalisP. gingivalis LPS (LPS (dd), FITC), FITC-- P. gingivalisP. gingivalis PGN (PGN (ee), FITC), FITC--E. coli E. coli LPS (LPS (ff), and FITC), and FITC--S. aureus S. aureus PGN (PGN (gg) ) of of gingival gingival epithelial tissuesepithelial tissues ((WT or WT or overexpressing CXADRoverexpressing CXADR)) infected with infected with P. gingivalisP. gingivalis. . ThreeThree--dimensional dimensional tissues on culture inserttissues on culture insertss were were infected with infected with P. gingivalisP. gingivalis and FITCand FITC-labeled labeled tracer in the upper compartment. Following 30 min of incubation, the tracer in the upper compartment. Following $30 \mathrm{~min}$ of incubation, the transmission of transmission of tracer from the upper compartment to the lower compartment was analyzed tracer from the upper compartment to the lower compartment was analyzed by by spectrometryspectrometry. Results are expressed as fold. Results are expressed as fold change relative to uninfectedchange relative to uninfected WT WT cells andcells and are the meanare the meanss \pm SD of \pm SD of seven seven technical replicates. ${ }^{*}$ technical replicates. ${ }^{*}{ } \mathrm{p}<0.05$, onep $<0.05$, onetailedtailed tt test (closed test (closed testing procedure).testing procedure). 


\section{Commensal bacteria}

$P$. gingivalis

- Gingipains

LPS

- PGN

JAM1

CXADR

Gingival epithelium
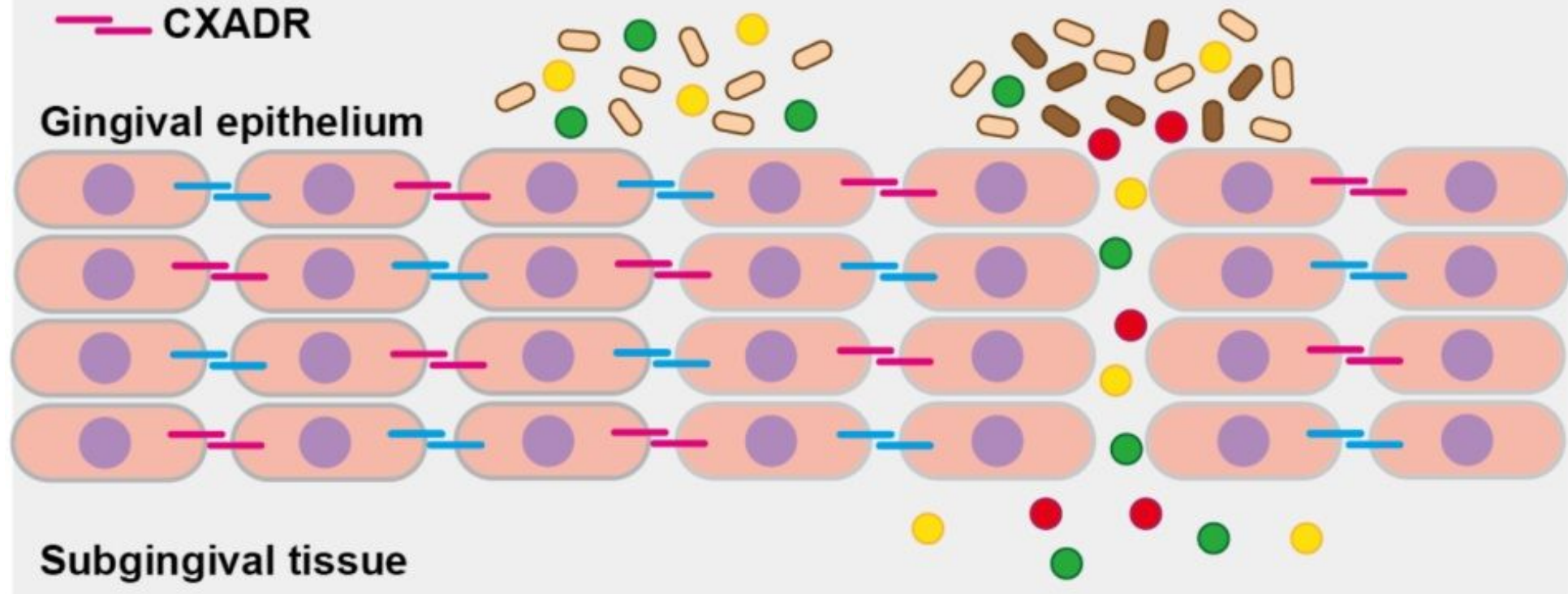

Figure 11

Proposed model of how P. gingivalis gingipains send bacterial virulence factors through the gingival epithelium. In gingival epithelial tissues CXADR ( and JAM1 (are not interdependent on the permeability. P. gingivalis gingipains degrade CXADR and JAM1, which increases the permeability of gingival epithelium to gingipains and other factors .Subsequently, gingipains are transferred to the deeper epithelium to fu rther degrade CXADR and JAM1, which allows LPS and PGN to penetrate the gingival epithelium and reach subepithelial tissues. Finally, gingipains, LPS, and PGN induce inflammation in gingival tissues.

\section{Supplementary Files}

This is a list of supplementary files associated with this preprint. Click to download.

- TakeuchietalSupplementaryInformationver2.pdf 